\title{
Complete semi-analytical treatment of weakly singular integrals on planar triangles via the direct evaluation method
}

\author{
Athanasios G. Polimeridis*, $\dagger$ and Juan R. Mosig \\ Laboratory of Electromagnetics and Acoustics (LEMA), Ecole Polytechnique Fédérale de Lausanne (EPFL), \\ CH-1015 Lausanne, Switzerland
}

\begin{abstract}
SUMMARY
A complete semi-analytical treatment of the four-dimensional (4-D) weakly singular integrals over coincident, edge adjacent and vertex adjacent triangles, arising in the Galerkin discretization of mixed potential integral equation formulations, is presented. The overall analysis is based on the direct evaluation method, utilizing a series of coordinate transformations, together with a re-ordering of the integrations, in order to reduce the dimensionality of the original 4-D weakly singular integrals into, respectively, 1-D, 2-D and $3-\mathrm{D}$ numerical integrations of smooth functions. The analytically obtained final formulas can be computed by using typical library routines for Gauss quadrature readily available in the literature. A comparison of the proposed method with singularity subtraction, singularity cancellation and fully numerical methods, often used to tackle the multi-dimensional singular integrals evaluation problem, is provided through several numerical examples, which clearly highlights the superior accuracy and efficiency of the direct evaluation scheme. Copyright (C) 2010 John Wiley \& Sons, Ltd.
\end{abstract}

Received 1 April 2009; Accepted 24 January 2010

KEY WORDS: Galerkin inner products; method of moments; mixed potential integral equations; weakly singular integrals

\section{INTRODUCTION}

Integral equation-based algorithms stand as one of the most accurate and efficient tools for tackling a wide variety of problems in various fields of applied physics and engineering. In computational electromagnetics, more specifically, the mixed potential integral equation formulation seems to have aroused a lot of attention over the last years, especially when dealing with electromagnetic scattering and radiation problems from an arbitrary perfect conducting surface [1,2]. The numerical solution of the surface mixed potential integral equations via the method of moments [3], using

\footnotetext{
*Correspondence to: Athanasios G. Polimeridis, Laboratory of Electromagnetics and Acoustics (LEMA), Ecole Polytechnique Fédérale de Lausanne (EPFL), CH-1015 Lausanne, Switzerland.

${ }^{\dagger}$ E-mail: athanasios.polymeridis@epfl.ch

Copyright (C) 2010 John Wiley \& Sons, Ltd.
} 
the linear Rao-Wilton-Glisson basis functions [1], calls for the computation of the following four-dimensional (4-D) weakly singular integrals:

$$
I_{p, q}^{\text {sing }}=\int_{E_{P}} \zeta_{p} \int_{E_{Q}} g\left(\boldsymbol{r}, \boldsymbol{r}^{\prime}\right) \zeta_{q}^{\prime} \mathrm{d} A_{Q} \mathrm{~d} A_{P}, \quad p, q=1,2,3
$$

and

$$
I_{S}^{\text {sing }}=\int_{E_{P}} \int_{E_{Q}} g\left(\boldsymbol{r}, \boldsymbol{r}^{\prime}\right) \mathrm{d} A_{Q} \mathrm{~d} A_{P},
$$

where the two triangular elements $E_{P}$ and $E_{Q}$ may coincide (coincident integration), share a common edge (edge adjacent integration), or share a common vertex (vertex adjacent integration). $A_{P}, A_{Q}$ are the associated triangular surfaces, $g\left(\boldsymbol{r}, \boldsymbol{r}^{\prime}\right)=\mathrm{e}^{-\mathrm{j} k R} / R$ is the free-space Green's function, $R=\left|\boldsymbol{r}-\boldsymbol{r}^{\prime}\right|$ is the distance function, $k$ is the wavenumber of the medium and $\zeta_{p}, \zeta_{q}^{\prime}$ are simplex coordinates associated with arbitrary vertices of the triangular elements.

In general, weakly singular integrals are treated by using mainly the singularity subtraction method [4-14] or the singularity cancellation method [15-25]. Despite their widespread usage, both singularity subtraction and singularity cancellation methods fail to meet the requirements for an accurate and efficient numerical integration of weakly singular integrals, as it will be demonstrated by the numerical examples in next sections. On the other hand, some very promising new methods have appeared in the literature which seem to outperform the traditional techniques. In Reference [26], for instance, a method originated in the context of mechanics, which utilizes a series of coordinate transformations followed by an appropriate Duffy transform [27], is presented. Similarly, a direct approach for the evaluation of hyper-singular static surface integrals has been presented in References [28, 29]. Moreover, the later direct evaluation method was generalized by the first author for the case of the weakly singular integrals (1) and (2) over coincident triangular elements [30]. In this paper, a further extension of the aforementioned method for all the weakly singular integrals is presented. With the final formulas derived herein, the semi-analytical treatment of weakly singular integrals on planar triangles via the direct evaluation method can be considered as complete. Apart from the weakly singular case, the strongly singular and hyper-singular integrals (derivatives of Green's functions) stand as a very challenging quest and their detailed treatment is a matter of future investigation.

In the following section, we outline the fundamental properties of the equilateral triangle parameter space, which is used for the transformation of the original triangles to the equilateral master triangles. In Section 3, the direct evaluation method for the self-term integrals (coincident integration) is presented in detail. In Sections 4 and 5, the final formulas for the edge adjacent and vertex adjacent integration are derived and, finally, in Section 6 a comparison of the proposed method with three other competing techniques (the singularity subtraction method, the singularity cancellation method and a fully numerical method) is presented in terms of accuracy and efficiency. The results reveal the superior overall performance of the direct evaluation scheme.

\section{EQUILATERAL TRIANGLE PARAMETER SPACE}

The first step of the direct evaluation method is to introduce an appropriate parameter space, a procedure that is very common in the evaluation of multi-dimensional integrals. In this paper, 
following the procedure described in Reference [28], an equilateral parameter space $\{\eta, \xi\}$, where $-1 \leqslant \eta \leqslant 1,0 \leqslant \xi \leqslant \sqrt{3}(1-|\eta|)$, will be employed. This specific choice of parameter space is rather convenient for executing the coincident and the edge adjacent integration, due to its symmetry. The simplex coordinates in the new parametric space are given by

$$
\zeta_{1}=\frac{\sqrt{3}(1-\eta)-\xi}{2 \sqrt{3}}, \quad \zeta_{2}=\frac{\sqrt{3}(1+\eta)-\xi}{2 \sqrt{3}}, \quad \zeta_{3}=\frac{\xi}{\sqrt{3}},
$$

while the governing transformation matrices are as follows:

$$
\boldsymbol{r}=\left[\begin{array}{l}
x \\
y \\
z
\end{array}\right]=\left[\begin{array}{c}
\frac{x_{2}+x_{1}}{2} \\
\frac{y_{2}+y_{1}}{2} \\
\frac{z_{2}+z_{1}}{2}
\end{array}\right]+\left[\begin{array}{cc}
\frac{x_{2}-x_{1}}{2} & \frac{2 x_{3}-x_{1}-x_{2}}{2 \sqrt{3}} \\
\frac{y_{2}-y_{1}}{2} & \frac{2 y_{3}-y_{1}-y_{2}}{2 \sqrt{3}} \\
\frac{z_{2}-z_{1}}{2} & \frac{2 z_{3}-z_{1}-z_{2}}{2 \sqrt{3}}
\end{array}\right]\left[\begin{array}{l}
\eta \\
\xi
\end{array}\right]=[\boldsymbol{A}]+[\boldsymbol{Q}]\left[\begin{array}{l}
\eta \\
\xi
\end{array}\right]
$$

and the Jacobian is just a constant and equals $J=A / \sqrt{3}$, where $A$ is the area of the original triangle. The weakly singular integrals in Equations (1), (2) can be evaluated as a linear combination of the following terms:

$$
I_{p, q}=\int_{-1}^{1} \mathrm{~d} \eta \int_{0}^{\xi(\eta)} \psi_{p}(\eta, \xi) \mathrm{d} \xi \int_{-1}^{1} \mathrm{~d} \eta^{\prime} \int_{0}^{\xi\left(\eta^{\prime}\right)} \psi_{q}\left(\eta^{\prime}, \xi^{\prime}\right) \frac{\mathrm{e}^{-\mathrm{j} k R}}{R} \mathrm{~d} \xi^{\prime}, \quad p, q=1,2,3,
$$

where $\xi(\eta)=\sqrt{3}(1-|\eta|)$. Moreover,

$$
\psi_{1}(\eta, \xi)=1, \quad \psi_{2}(\eta, \xi)=\eta, \quad \psi_{3}(\eta, \xi)=\xi
$$

and $R$ is a function of all six nodal coordinates (for the two original triangles) as well as the four variables (two for the inner integral $E_{Q}$ and two for the outer integral $E_{P}$ ) of the equilateral triangle parametric space.

More specifically, the weakly singular integrals (1) and (2) are derived with the help of Equations (3)-(5), leading to the following expressions:

$$
\begin{aligned}
& I_{s}^{\text {sing }}=\left(J_{p} J_{q}\right) I_{1,1}, \\
& I_{1,1}^{\text {sing }}=\frac{J_{p} J_{q}}{12}\left[3 I_{1,1}-3 I_{1,2}-\sqrt{3} I_{1,3}-3 I_{2,1}+3 I_{2,2}+\sqrt{3} I_{2,3}-\sqrt{3} I_{3,1}+\sqrt{3} I_{3,2}+I_{3,3}\right], \\
& I_{1,2}^{\text {sing }}=\frac{J_{p} J_{q}}{12}\left[3 I_{1,1}+3 I_{1,2}-\sqrt{3} I_{1,3}-3 I_{2,1}-3 I_{2,2}+\sqrt{3} I_{2,3}-\sqrt{3} I_{3,1}-\sqrt{3} I_{3,2}+I_{3,3}\right], \\
& I_{1,3}^{\text {sing }}=\frac{J_{p} J_{q}}{6}\left[\sqrt{3} I_{1,3}-\sqrt{3} I_{2,3}-I_{3,3}\right], \\
& I_{2,1}^{\text {sing }}=\frac{J_{p} J_{q}}{12}\left[3 I_{1,1}-3 I_{1,2}-\sqrt{3} I_{1,3}+3 I_{2,1}-3 I_{2,2}-\sqrt{3} I_{2,3}-\sqrt{3} I_{3,1}+\sqrt{3} I_{3,2}+I_{3,3}\right], \\
& I_{2,2}^{\text {sing }}=\frac{J_{p} J_{q}}{12}\left[3 I_{1,1}+3 I_{1,2}-\sqrt{3} I_{1,3}+3 I_{2,1}+3 I_{2,2}-\sqrt{3} I_{2,3}-\sqrt{3} I_{3,1}-\sqrt{3} I_{3,2}+I_{3,3}\right],
\end{aligned}
$$




$$
\begin{aligned}
& I_{2,3}^{\text {sing }}=\frac{J_{p} J_{q}}{6}\left[\sqrt{3} I_{1,3}+\sqrt{3} I_{2,3}-I_{3,3}\right], \\
& I_{3,1}^{\text {sing }}=\frac{J_{p} J_{q}}{6}\left[\sqrt{3} I_{3,1}-\sqrt{3} I_{3,2}-I_{3,3}\right], \\
& I_{3,2}^{\text {sing }}=\frac{J_{p} J_{q}}{6}\left[\sqrt{3} I_{3,1}+\sqrt{3} I_{3,2}-I_{3,3}\right], \\
& I_{3,3}^{\text {sing }}=\frac{J_{p} J_{q}}{3} I_{3,3},
\end{aligned}
$$

where $J_{p}$ and $J_{q}$ are the constant Jacobians of the transformation from the original triangles to the equilateral parameter space master triangles.

\subsection{Coincident triangles}

In the case of the so-called self-term evaluation (or coincident integration) the outer and inner triangles coincide, i.e. $E_{P} \equiv E_{Q}$. Hence, it is easy to prove that the distance function can be written as

$$
R=\left|\boldsymbol{r}_{\boldsymbol{P}}-\boldsymbol{r}_{\boldsymbol{Q}}\right|=\sqrt{\alpha_{c_{1}}\left(\eta-\eta^{\prime}\right)^{2}+\alpha_{c_{2}}\left(\eta-\eta^{\prime}\right)\left(\xi-\xi^{\prime}\right)+\alpha_{c_{3}}\left(\xi-\xi^{\prime}\right)^{2}},
$$

where

$$
\alpha_{c_{1}}=\frac{1}{4}\left|\boldsymbol{r}_{1}-\boldsymbol{r}_{2}\right|^{2}, \quad \alpha_{c_{2}}=\frac{1}{2 \sqrt{3}}\left(\boldsymbol{r}_{1}-\boldsymbol{r}_{2}\right) \cdot\left(\boldsymbol{r}_{1}+\boldsymbol{r}_{2}-2 \boldsymbol{r}_{3}\right), \quad \alpha_{c_{3}}=\frac{1}{12}\left|\boldsymbol{r}_{1}+\boldsymbol{r}_{2}-2 \boldsymbol{r}_{3}\right|^{2}
$$

and $\boldsymbol{r}_{i}=\left(x_{i}, y_{i}, z_{i}\right), i=1,2,3$ are the vertices of the triangular element.

\subsection{Edge adjacent triangles}

In the case of triangular elements sharing a common edge (edge adjacent integration), we need to orient the elements so that the shared edge is defined by $\xi=0$ for $E_{P}$, and $\xi^{\prime}=0$ for $E_{Q}$, and the singularity is characterized by $\eta+\eta^{\prime}=\xi=\xi^{\prime}=0$. The position vectors are given by $E_{P}$ : $\left(\boldsymbol{r}_{1 p}, \boldsymbol{r}_{2 p}, \boldsymbol{r}_{3 p}\right) \equiv\left(\boldsymbol{r}_{1}, \boldsymbol{r}_{2}, \boldsymbol{r}_{3}\right), E_{Q}:\left(\boldsymbol{r}_{1 q}, \boldsymbol{r}_{2 q}, \boldsymbol{r}_{3 q}\right) \equiv\left(\boldsymbol{r}_{2}, \boldsymbol{r}_{1}, \boldsymbol{r}_{4}\right)$ and, again, the distance function in the new parametric space can be derived as follows:

$$
\begin{aligned}
R= & \left|\boldsymbol{r}_{\boldsymbol{P}}-\boldsymbol{r}_{\boldsymbol{Q}}\right|=\left(\left|\boldsymbol{\alpha}_{e_{2}}\right|^{2} \xi^{2}+2 \xi\left[\left(\boldsymbol{\alpha}_{e_{2}} \cdot \boldsymbol{\alpha}_{e_{3}}\right) \xi^{\prime}+\left(\boldsymbol{\alpha}_{e_{1}} \cdot \boldsymbol{\alpha}_{e_{2}}\right)\left(\eta+\eta^{\prime}\right)\right]\right. \\
& \left.+\left|\boldsymbol{\alpha}_{e_{1}}\right|^{2}\left(\eta+\eta^{\prime}\right)^{2}+\left|\boldsymbol{\alpha}_{e_{3}}\right|^{2} \xi^{\prime 2}+2\left(\boldsymbol{\alpha}_{e_{1}} \cdot \boldsymbol{\alpha}_{e_{3}}\right)\left(\eta+\eta^{\prime}\right) \xi^{\prime}\right)^{1 / 2}
\end{aligned}
$$

where

$$
\alpha_{e_{1}}=\frac{\boldsymbol{r}_{2}-\boldsymbol{r}_{1}}{2}, \quad \alpha_{e_{2}}=\frac{2 \boldsymbol{r}_{3}-\boldsymbol{r}_{1}-\boldsymbol{r}_{2}}{2 \sqrt{3}}, \quad \alpha_{e_{3}}=-\frac{2 \boldsymbol{r}_{4}+\boldsymbol{r}_{1}-\boldsymbol{r}_{2}}{2 \sqrt{3}}
$$

\subsection{Vertex adjacent triangles}

Finally, in the case of triangular elements sharing a common vertex (vertex adjacent integration), we need to orient the elements so that the singular point (shared vertex) is $\eta=-1$ and $\eta^{\prime}=-1$. 
Again, the position vectors are given by $E_{P}:\left(\boldsymbol{r}_{1 p}, \boldsymbol{r}_{2 p}, \boldsymbol{r}_{3 p}\right) \equiv\left(\boldsymbol{r}_{1}, \boldsymbol{r}_{2}, \boldsymbol{r}_{3}\right)$ and $E_{Q}:\left(\boldsymbol{r}_{1 q}, \boldsymbol{r}_{2 q}, \boldsymbol{r}_{3 q}\right) \equiv$ $\left(\boldsymbol{r}_{1}, \boldsymbol{r}_{4}, \boldsymbol{r}_{5}\right)$. The distance function in the new equilateral triangle parameter space space is given by

$$
R=\left|\boldsymbol{r}_{\boldsymbol{P}}-\boldsymbol{r}_{\boldsymbol{Q}}\right|=\left|\boldsymbol{\alpha}_{v_{0}}+\boldsymbol{\alpha}_{v_{1}} \eta+\boldsymbol{\alpha}_{v_{2}} \xi+\boldsymbol{\alpha}_{v_{3}} \eta^{\prime}+\boldsymbol{\alpha}_{v_{4}} \xi^{\prime}\right|,
$$

where

$$
\begin{aligned}
& \boldsymbol{\alpha}_{v_{0}}=\frac{\boldsymbol{r}_{2}-\boldsymbol{r}_{4}}{2}, \quad \boldsymbol{\alpha}_{v_{1}}=\frac{\boldsymbol{r}_{2}-\boldsymbol{r}_{1}}{2}, \quad \boldsymbol{\alpha}_{v_{2}}=\frac{2 \boldsymbol{r}_{3}-\boldsymbol{r}_{1}-\boldsymbol{r}_{2}}{2 \sqrt{3}}, \\
& \boldsymbol{\alpha}_{v_{3}}=\frac{\boldsymbol{r}_{4}-\boldsymbol{r}_{1}}{2}, \quad \boldsymbol{\alpha}_{v_{4}}=-\frac{2 \boldsymbol{r}_{5}+\boldsymbol{r}_{1}-\boldsymbol{r}_{4}}{2 \sqrt{3}} .
\end{aligned}
$$

\section{COINCIDENT INTEGRATION}

\subsection{First analytical integration}

To begin with the inner integration, a polar coordinate system centered at the point $(\eta, \xi)$ of the outer integration is defined,

$$
\eta^{\prime}-\eta=\rho \cos (\theta), \quad \xi^{\prime}-\xi=\rho \sin (\theta),
$$

as illustrated in Figure 1. Generally, polar coordinate transformations have been widely used in the singularity cancellation methods, as the Jacobian of the transformation cancels the weak singularity of the integrand. The distance function becomes $R=\left|\boldsymbol{r}-\boldsymbol{r}^{\prime}\right|=\rho \cdot \alpha(\theta)$, where

$$
\alpha(\theta)=\sqrt{\alpha_{c_{1}} \cos ^{2}(\theta)+\alpha_{c_{2}} \cos (\theta) \sin (\theta)+\alpha_{c_{3}} \sin ^{2}(\theta)} .
$$

Because the expression for the upper limit of $\rho, \rho_{\mathrm{L}}(\theta)$, is different as $\theta$ traverses each edge, the $(\rho, \theta)$ integration must be split into three subtriangles. In the following, only the calculation for the lower subtriangle with the edge $\xi^{\prime}=0$ will be presented. Exploiting the symmetry of the equilateral parameter space, the remaining two subtriangles are handled by rotating the element

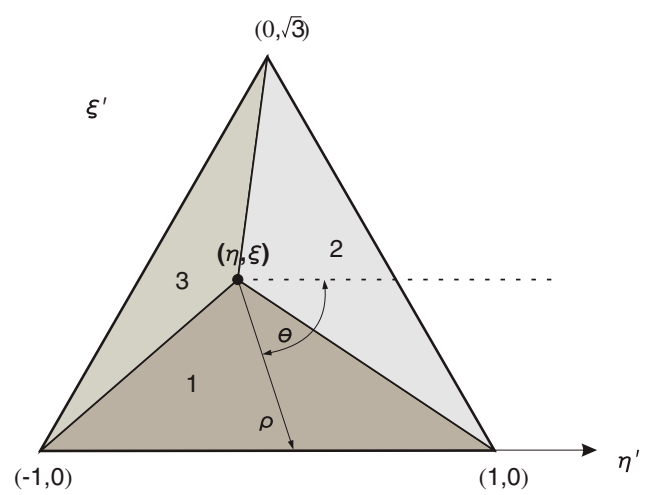

Figure 1. First polar coordinate transformation $\left\{\eta^{\prime}, \xi^{\prime}\right\} \rightarrow\{\rho, \theta\}$ and splitting of the master triangle into three subtriangles. 
and employing the formulas for the lower subtriangle, as it will be shown next. For the lower subtriangle, the integration limits are $0 \leqslant \rho \leqslant \rho_{\mathrm{L}}$ and $\Theta_{1} \leqslant \theta \leqslant \Theta_{2}$, where

$$
\rho_{\mathrm{L}}=-\frac{\xi}{\sin (\theta)}, \quad \Theta_{1}=-\frac{\pi}{2}-\tan ^{-1}\left(\frac{1+\eta}{\xi}\right), \quad \Theta_{2}=-\frac{\pi}{2}+\tan ^{-1}\left(\frac{1-\eta}{\xi}\right) .
$$

Hence, the inner integration of Equation (5) becomes

$$
I_{q}^{\mathrm{in}}(\eta, \xi)=\int_{\Theta_{1}}^{\Theta_{2}} \int_{0}^{\rho_{\mathrm{L}}} \psi_{q}(\eta, \xi, \rho, \theta) \frac{\mathrm{e}^{-\mathrm{j} k \rho \alpha(\theta)}}{\alpha(\theta)} \mathrm{d} \rho \mathrm{d} \theta=\int_{\Theta_{1}}^{\Theta_{2}} I_{q}^{\rho}(\eta, \xi, \theta) \mathrm{d} \theta,
$$

where the term

$$
I_{q}^{\rho}(\eta, \xi, \theta)=\int_{0}^{\rho_{\mathrm{L}}} \psi_{q}(\eta, \xi, \rho, \theta) \frac{\mathrm{e}^{-\mathrm{j} k \rho \alpha(\theta)}}{\alpha(\theta)} \mathrm{d} \rho
$$

can be analytically evaluated. The integral for the lower subtriangle is given by:

$$
I_{p, q}^{\mathrm{sub}_{1}}=\int_{-1}^{1} \mathrm{~d} \eta \int_{0}^{\xi(\eta)} \psi_{p}(\eta, \xi) \mathrm{d} \xi \int_{\Theta_{1}}^{\Theta_{2}} I_{q}^{\rho}(\eta, \xi, \theta) \mathrm{d} \theta .
$$

Clearly, the first analytic integration is sufficient to cancel the weakly singular behavior of the integrals and the remaining 3-D integral can be computed via a relatively simple scheme of Gauss quadrature product rule. As already stated, however, the overall performance of such a method does not meet the requirements for an accurate and efficient evaluation of weakly singular integrals. Hence, in the following, we will continue with the reduction in the dimensionality of the original triangle and, ultimately, derive 1-D formulas that provide greater accuracy with less computational effort.

\subsection{Second analytical integration}

In order to proceed to the second analytical integration, we have to introduce, first, the variable $t$, $-1 \leqslant t \leqslant 1$, via

$$
\theta=-\frac{\pi}{2}+\tan ^{-1}\left(\frac{t-\eta}{\xi}\right), \quad \frac{\mathrm{d} \theta}{\mathrm{d} t}=\frac{\xi}{\xi^{2}+(t-\eta)^{2}}=F(\eta, \xi, t),
$$

which results in $\rho_{\mathrm{L}}=\sqrt{\xi^{2}+(t-\eta)^{2}}$. Interchanging the order of integration, (28) becomes

$$
I_{p, q}^{\mathrm{sub}_{1}}=\int_{-1}^{1} \mathrm{~d} \eta \int_{-1}^{1} \mathrm{~d} t \int_{0}^{\xi(\eta)} \psi_{p}(\eta, \xi) I_{q}^{\rho}(\eta, \xi, t) F(\eta, \xi, t) \mathrm{d} \xi .
$$

Next, a new polar coordinate system $\{\Lambda, \Psi\}$ replaces $\{t, \xi\}$,

$$
t=\Lambda \cos (\Psi)+\eta, \quad \xi=\Lambda \sin (\Psi) .
$$

With the two changes of variables, $\theta \rightarrow t$ and $\{t, \xi\} \rightarrow\{\Lambda$, $\Psi\}$, we get the following:

$$
\cos (\theta) \rightarrow \cos (\Psi), \quad \sin (\theta) \rightarrow-\sin (\Psi), \quad \rho_{\mathrm{L}} \rightarrow \Lambda, \quad F \rightarrow \frac{\sin (\Psi)}{\Lambda}
$$




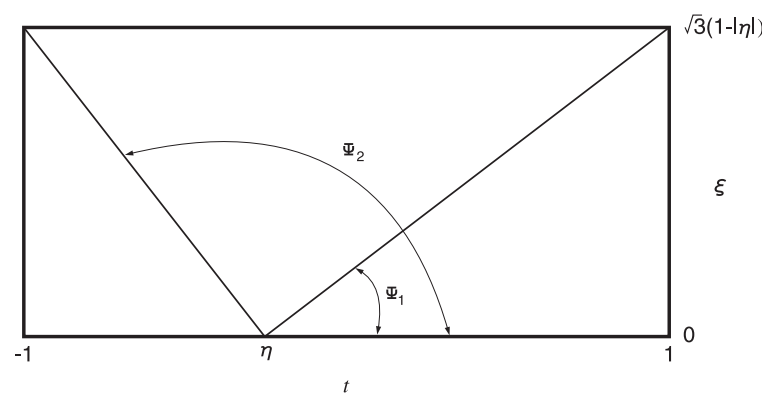

Figure 2. Geometry of the second polar coordinate transformation, $\{t, \xi\} \rightarrow\{\Lambda, \Psi\}$.

We have to note that the $\{t, \xi\}$ domain is a rectangle (Figure 2), and integrating over $\{\Lambda, \Psi\}$ will necessitate a decomposition into three subdomains: $\Psi_{0} \leqslant \Psi \leqslant \Psi_{1}, \Psi_{1} \leqslant \Psi \leqslant \Psi_{2}$ and $\Psi_{2} \leqslant \Psi \leqslant \Psi_{3}$, where $\Psi_{0}=0, \Psi_{1}=\tan ^{-1}[\xi(\eta) /(1-\eta)], \Psi_{2}=\pi-\tan ^{-1}[\xi(\eta) /(1+\eta)]$ and $\Psi_{3}=\pi$. The integral with respect to $\Lambda$ can be evaluated analytically, and Equation (28) becomes

$$
I_{p, q}^{\mathrm{sub}_{1}}=\sum_{m=0}^{2} \int_{-1}^{1} \mathrm{~d} \eta \int_{\Psi_{m}}^{\Psi_{m+1}} \sin (\Psi) I_{p, q}^{\Lambda}(\eta, \Psi) \mathrm{d} \Psi,
$$

where

$$
I_{p, q}^{\Lambda}(\eta, \Psi)=\int_{0}^{\Lambda_{L}} \psi_{p}(\eta, \Lambda, \Psi) I_{q}^{\rho}(\eta, \Lambda, \Psi) \mathrm{d} \Lambda
$$

and the limit $\Lambda_{L}$ for the integral over $\Lambda$ depends upon the particular subdomain $(m=0,1,2)$ being considered, as will be shown next.

\subsection{Third analytical integration}

The weakly singular integral (5) has been reduced to an integration over $\{\eta, \Psi\}$ with the $\Psi$ integral decomposed into three subintegrals. The main objective is to re-order the integral and integrate with respect to $\eta$ analytically, and thus each subintegral has to be examined separately. Also, we split the $\eta$ integral as $-1<\eta<0$ and $0 \leqslant \eta<1$.

3.3.1. Integration over region $\eta \geqslant 0$. In this case, the subdivision of the integral with respect to $\Psi$ and the limits of the integration with respect to $\Lambda$ are (Figure 3(a))

$$
\begin{array}{cl}
0 & \leqslant \Psi \leqslant \frac{\pi}{3}, \quad \Lambda_{L}=(1-\eta) / \cos (\Psi), \\
\frac{\pi}{3} & \leqslant \Psi \leqslant \Psi_{\eta}, \quad \Lambda_{L}=\sqrt{3}(1-\eta) / \sin (\Psi), \\
\Psi_{\eta} & \leqslant \Psi \leqslant \pi, \quad \Lambda_{L}=-(1+\eta) / \cos (\Psi),
\end{array}
$$

where $\Psi_{\eta} \equiv \Psi_{2}=\pi-\tan ^{-1}(\sqrt{3}(1-\eta) /(1+\eta))$. 


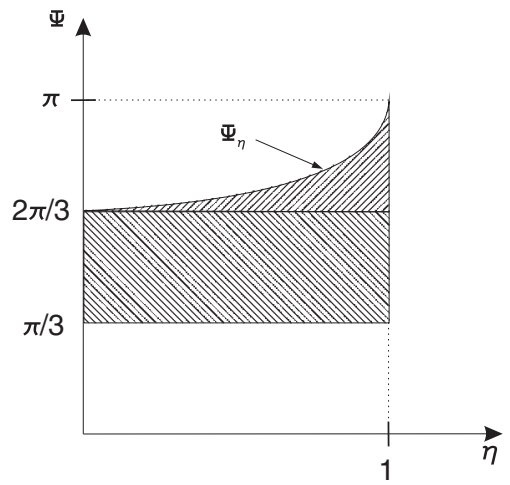

(a)

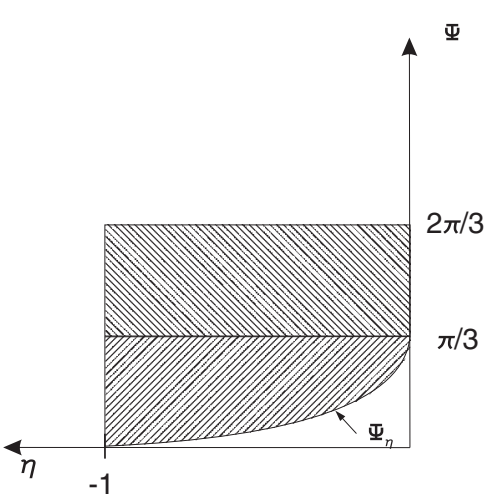

(b)

Figure 3. Geometry of the parametric space $\{\eta, \Psi\}$ : (a) $\eta>0$ and (b) $\eta<0$.

Interchanging the order of integration, and subsequently evaluating the integral with respect to $\eta$ we get the following three integrals with respect to $\Psi$ :

$$
\begin{aligned}
\int_{0}^{1} \mathrm{~d} \eta \int_{0}^{\pi / 3} \sin (\Psi) I_{p, q}^{\Lambda}(\eta, \Psi) \mathrm{d} \Psi= & \int_{0}^{\pi / 3} \sin (\Psi) \mathrm{d} \Psi \int_{0}^{1} I_{p, q}^{\Lambda}(\eta, \Psi) \mathrm{d} \eta=\int_{0}^{\pi / 3} \Phi_{p, q}^{a}(\Psi) \mathrm{d} \Psi \\
\int_{0}^{1} \mathrm{~d} \eta \int_{\pi / 3}^{\Psi_{2}} \sin (\Psi) I_{p, q}^{\Lambda}(\eta, \Psi) \mathrm{d} \Psi= & \int_{\pi / 3}^{2 \pi / 3} \sin (\Psi) \mathrm{d} \Psi \int_{0}^{1} I_{p, q}^{\Lambda}(\eta, \Psi) \mathrm{d} \eta \\
& +\int_{2 \pi / 3}^{\pi} \sin (\Psi) \mathrm{d} \Psi \int_{\alpha}^{1} I_{p, q}^{\Lambda}(\eta, \Psi) \mathrm{d} \eta \\
= & \int_{\pi / 3}^{2 \pi / 3} \Phi_{p, q}^{b}(\Psi) \mathrm{d} \Psi+\int_{2 \pi / 3}^{\pi} \Phi_{p, q}^{c}(\Psi) \mathrm{d} \Psi \\
\int_{0}^{1} \mathrm{~d} \eta \int_{\Psi_{2}}^{\pi} \sin (\Psi) I_{p, q}^{\Lambda}(\eta, \Psi) \mathrm{d} \Psi= & \int_{2 \pi / 3}^{\pi} \sin (\Psi) \mathrm{d} \Psi \int_{0}^{\alpha} I_{p, q}^{\Lambda}(\eta, \Psi) \mathrm{d} \eta=\int_{2 \pi / 3}^{\pi} \Phi_{p, q}^{d}(\Psi) \mathrm{d} \Psi
\end{aligned}
$$

where $\alpha$ is obtained by inverting the formula for $\Psi_{\eta}$,

$$
\alpha=\frac{1-\beta}{1+\beta}, \quad \beta=\tan (\pi-\Psi) / \sqrt{3} .
$$

The $\Phi_{p, q}^{a-d}$ functions result from the analytic integration with respect to $\eta$ multiplied with the sine function. 
3.3.2. Integration over region $\eta \leqslant 0$. Equally, for the case where $\eta \leqslant 0$, the procedures are essentially the same as above. Hence, the subdivision of the integral with respect to $\Psi$ leads to (Figure 3(b))

$$
\begin{array}{cl}
0 \leqslant \Psi \leqslant \Psi_{\eta}, & \Lambda_{L}=(1-\eta) / \cos (\Psi), \\
\Psi_{\eta} \leqslant \Psi \leqslant \frac{2 \pi}{3}, & \Lambda_{L}=\sqrt{3}(1+\eta) / \sin (\Psi), \\
\frac{2 \pi}{3} \leqslant \Psi \leqslant \pi, & \Lambda_{L}=-(1+\eta) / \cos (\Psi),
\end{array}
$$

where

$$
\Psi_{\eta}=\tan ^{-1}(\sqrt{3}(1+\eta) /(1-\eta))
$$

Again, by interchanging the order of integration and integrating analytically we get the following results:

$$
\begin{aligned}
& \int_{-1}^{0} \mathrm{~d} \eta \int_{0}^{\Psi_{\eta}} \sin (\Psi) I_{p, q}^{\Lambda}(\eta, \Psi) \mathrm{d} \Psi \\
& =\int_{0}^{\pi / 3} \sin (\Psi) \mathrm{d} \Psi \int_{\alpha}^{0} I_{p, q}^{\Lambda}(\eta, \Psi) \mathrm{d} \eta=\int_{0}^{\pi / 3} \Phi_{p, q}^{e}(\Psi) \mathrm{d} \Psi \\
& \int_{-1}^{0} \mathrm{~d} \eta \int_{\Psi_{\eta}}^{2 \pi / 3} \sin (\Psi) I_{p, q}^{\Lambda}(\eta, \Psi) \mathrm{d} \Psi \\
& =\int_{0}^{\pi / 3} \sin (\Psi) \mathrm{d} \Psi \int_{-1}^{\alpha} I_{p, q}^{\Lambda}(\eta, \Psi) \mathrm{d} \eta+\int_{\pi / 3}^{2 \pi / 3} \sin (\Psi) \mathrm{d} \Psi \int_{-1}^{0} I_{p, q}^{\Lambda}(\eta, \Psi) \mathrm{d} \eta \\
& =\int_{0}^{\pi / 3} \Phi_{p, q}^{f}(\Psi) \mathrm{d} \Psi+\int_{\pi / 3}^{2 \pi / 3} \Phi_{p, q}^{g}(\Psi) \mathrm{d} \Psi, \\
& \int_{-1}^{0} \mathrm{~d} \eta \int_{2 \pi / 3}^{\pi} \sin (\Psi) I_{p, q}^{\Lambda}(\eta, \Psi) \mathrm{d} \Psi \\
& =\int_{2 \pi / 3}^{\pi} \sin (\Psi) \mathrm{d} \Psi \int_{-1}^{0} I_{p, q}^{\Lambda}(\eta, \Psi) \mathrm{d} \eta=\int_{2 \pi / 3}^{\pi} \Phi_{p, q}^{h}(\Psi) \mathrm{d} \Psi
\end{aligned}
$$

where this time

$$
\alpha=-\frac{1-\beta}{1+\beta}, \quad \beta=\tan (\Psi) / \sqrt{3} .
$$

Finally, the weakly singular integral for the lower subtriangle has been reduced to the following 1-D smooth integral:

$$
\begin{aligned}
I_{p, q}^{\mathrm{sub}_{1}}= & \int_{0}^{\pi / 3}\left[\Phi_{p, q}^{a}(\Psi)+\Phi_{p, q}^{e}(\Psi)+\Phi_{p, q}^{f}(\Psi)\right] \mathrm{d} \Psi+\int_{\pi / 3}^{2 \pi / 3}\left[\Phi_{p, q}^{b}(\Psi)+\Phi_{p, q}^{g}(\Psi)\right] \mathrm{d} \Psi \\
& +\int_{2 \pi / 3}^{\pi}\left[\Phi_{p, q}^{c}(\Psi)+\Phi_{p, q}^{d}(\Psi)+\Phi_{p, q}^{h}(\Psi)\right] \mathrm{d} \Psi .
\end{aligned}
$$


The numerical integration of the remaining integral can easily be evaluated with a Gauss quadrature rule and as it will be shown later on, the arithmetic results converge to the exact value with a relatively small number of integration points.

\subsection{Final formulas}

Although the remaining two cases could be handled in exactly the same manner, this would require repeating the analysis above for the other two subtriangles. An alternate route, which clearly simplifies the implementation, is to exploit the symmetry of the equilateral parameter space. Hence, the remaining subtriangles are handled by rotating the master triangle and employing the formulas for the lower subtriangle. More specifically, the expressions for the second subtriangle are given by the following formula:

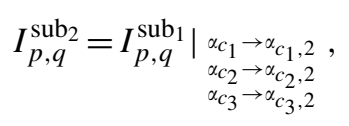

where

$$
\begin{aligned}
& \alpha_{c_{1}, 2}=\frac{1}{4} \alpha_{c_{1}}-\frac{\sqrt{3}}{4} \alpha_{c_{2}}+\frac{3}{4} \alpha_{c_{3}}, \\
& \alpha_{c_{2}, 2}=\frac{\sqrt{3}}{2} \alpha_{c_{1}}-\frac{1}{2} \alpha_{c_{2}}-\frac{\sqrt{3}}{2} \alpha_{c_{3}}, \\
& \alpha_{c_{3}, 2}=\frac{3}{4} \alpha_{c_{1}}+\frac{\sqrt{3}}{4} \alpha_{c_{2}}+\frac{1}{4} \alpha_{c_{3}} .
\end{aligned}
$$

Equally, for the third subtriangle the rotation leads to the following formula:

$$
I_{p, q}^{\operatorname{sub}_{3}}=I_{p, q}^{\operatorname{sub}_{1}} \mid \begin{aligned}
& \alpha_{c_{1} \rightarrow \alpha_{c_{1}}, 3}, \\
& \alpha_{c_{2} \rightarrow \alpha_{c_{2}}, 3}, \\
& \alpha_{c_{3} \rightarrow \alpha_{c_{3}, 3}}
\end{aligned}
$$

where

$$
\begin{aligned}
& \alpha_{c_{1}, 3}=\frac{1}{4} \alpha_{c_{1}}+\frac{\sqrt{3}}{4} \alpha_{c_{2}}+\frac{3}{4} \alpha_{c_{3}}, \\
& \alpha_{c_{2}, 3}=-\frac{\sqrt{3}}{2} \alpha_{c_{1}}-\frac{1}{2} \alpha_{c_{2}}+\frac{\sqrt{3}}{2} \alpha_{c_{3}}, \\
& \alpha_{c_{3}, 3}=\frac{3}{4} \alpha_{c_{1}}-\frac{\sqrt{3}}{4} \alpha_{c_{2}}+\frac{1}{4} \alpha_{c_{3}} .
\end{aligned}
$$

Next to the computation of the integrals for the subtriangles, we can proceed to the evaluation of the integrals in Equation (5). By rotating the master triangle and after a series of algebraic manipulations, the aforementioned integrals take the following form:

$$
\begin{aligned}
& I_{1,1}=I_{1,1}^{\mathrm{sub}_{1}}+I_{1,1}^{\mathrm{sub}_{2}}+I_{1,1}^{\mathrm{sub}_{3}}, \\
& I_{1,2}=I_{1,2}^{\mathrm{sub}_{1}}+\frac{1}{2} I_{1,1}^{\mathrm{sub}_{2}}-\frac{1}{2} I_{1,2}^{\mathrm{sub}_{2}}-\frac{\sqrt{3}}{2} I_{1,3}^{\mathrm{sub}_{2}}-\frac{1}{2} I_{1,1}^{\mathrm{sub}_{3}}-\frac{1}{2} I_{1,2}^{\mathrm{sub}_{3}}+\frac{\sqrt{3}}{2} I_{1,3}^{\mathrm{sub}_{3},} \\
& I_{1,3}=I_{1,3}^{\mathrm{sub}_{1}}+\frac{\sqrt{3}}{2} I_{1,1}^{\mathrm{sub}_{2}}+\frac{\sqrt{3}}{2} I_{1,2}^{\mathrm{sub}_{2}}-\frac{1}{2} I_{1,3}^{\mathrm{sub}_{2}}+\frac{\sqrt{3}}{2} I_{1,1}^{\mathrm{sub}_{3}}-\frac{\sqrt{3}}{2} I_{1,2}^{\mathrm{sub}_{3}}-\frac{1}{2} I_{1,3}^{\mathrm{sub}_{3},}
\end{aligned}
$$




$$
\begin{aligned}
& I_{2,1}=I_{2,1}^{\mathrm{sub}_{1}}+\frac{1}{2} I_{1,1}^{\mathrm{sub}_{2}}-\frac{1}{2} I_{2,1}^{\mathrm{sub}_{2}}-\frac{\sqrt{3}}{2} I_{3,1}^{\mathrm{sub}_{2}}-\frac{1}{2} I_{1,1}^{\mathrm{sub}_{3}}-\frac{1}{2} I_{2,1}^{\mathrm{sub}_{3}}+\frac{\sqrt{3}}{2} I_{3,1}^{\mathrm{sub}_{3}}, \\
& I_{2,2}=I_{2,2}^{\mathrm{sub}_{1}}+\frac{1}{4} I_{1,1}^{\mathrm{sub}_{2}}-\frac{1}{4} I_{1,2}^{\mathrm{sub}_{2}}-\frac{\sqrt{3}}{4} I_{1,3}^{\mathrm{sub}_{2}}-\frac{1}{4} I_{2,1}^{\mathrm{sub}_{2}}+\frac{1}{4} I_{2,2}^{\mathrm{sub}_{2}}+\frac{\sqrt{3}}{4} I_{2,3}^{\mathrm{sub}_{2}} \\
& -\frac{\sqrt{3}}{4} I_{3,1}^{\mathrm{sub}_{2}}+\frac{\sqrt{3}}{4} I_{3,2}^{\mathrm{sub}_{2}}+\frac{3}{4} I_{3,3}^{\mathrm{sub}_{2}}+\frac{1}{4} I_{1,1}^{\mathrm{sub}_{3}}+\frac{1}{4} I_{1,2}^{\mathrm{sub}_{3}}-\frac{\sqrt{3}}{4} I_{1,3}^{\mathrm{sub}_{3}}+\frac{1}{4} I_{2,1}^{\mathrm{sub}_{3}} \\
& +\frac{1}{4} I_{2,2}^{\mathrm{sub}_{3}}-\frac{\sqrt{3}}{4} I_{2,3}^{\mathrm{sub}_{3}}-\frac{\sqrt{3}}{4} I_{3,1}^{\mathrm{sub}_{3}}-\frac{\sqrt{3}}{4} I_{3,2}^{\mathrm{sub}_{3}}+\frac{3}{4} I_{3,3}^{\mathrm{sub}_{3},}, \\
& I_{2,3}=I_{2,3}^{\mathrm{sub}_{1}}+\frac{\sqrt{3}}{4} I_{1,1}^{\mathrm{sub}_{2}}+\frac{\sqrt{3}}{4} I_{1,2}^{\mathrm{sub}_{2}}-\frac{1}{4} I_{1,3}^{\mathrm{sub}_{2}}-\frac{\sqrt{3}}{4} I_{2,1}^{\mathrm{sub}_{2}}-\frac{\sqrt{3}}{4} I_{2,2}^{\mathrm{sub}_{2}}+\frac{1}{4} I_{2,3}^{\mathrm{sub}_{2}} \\
& -\frac{3}{4} I_{3,1}^{\mathrm{sub}_{2}}-\frac{3}{4} I_{3,2}^{\mathrm{sub}_{2}}+\frac{\sqrt{3}}{4} I_{3,3}^{\mathrm{sub}_{2}}-\frac{\sqrt{3}}{4} I_{1,1}^{\mathrm{sub}_{3}}+\frac{\sqrt{3}}{4} I_{1,2}^{\mathrm{sub}_{3}}+\frac{1}{4} I_{1,3}^{\mathrm{sub}_{3}}-\frac{\sqrt{3}}{4} I_{2,1}^{\mathrm{sub}_{3}} \\
& +\frac{\sqrt{3}}{4} I_{2,2}^{\mathrm{sub}_{3}}+\frac{1}{4} I_{2,3}^{\mathrm{sub}_{3}}+\frac{3}{4} I_{3,1}^{\mathrm{sub}_{3}}-\frac{3}{4} I_{3,2}^{\mathrm{sub}_{3}}-\frac{\sqrt{3}}{4} I_{3,3}^{\mathrm{sub}_{3}}, \\
& I_{3,1}=I_{3,1}^{\mathrm{sub}_{1}}+\frac{\sqrt{3}}{2} I_{1,1}^{\mathrm{sub}_{2}}+\frac{\sqrt{3}}{2} I_{2,1}^{\mathrm{sub}_{2}}-\frac{1}{2} I_{3,1}^{\mathrm{sub}_{2}}+\frac{\sqrt{3}}{2} I_{1,1}^{\mathrm{sub}_{3}}-\frac{\sqrt{3}}{2} I_{2,1}^{\mathrm{sub}_{3}}-\frac{1}{2} I_{3,1}^{\mathrm{sub}_{3}}, \\
& I_{3,2}=I_{3,2}^{\mathrm{sub}_{1}}+\frac{\sqrt{3}}{4} I_{1,1}^{\mathrm{sub}_{2}}-\frac{\sqrt{3}}{4} I_{1,2}^{\mathrm{sub}_{2}}-\frac{3}{4} I_{1,3}^{\mathrm{sub}_{2}}+\frac{\sqrt{3}}{4} I_{2,1}^{\mathrm{sub}_{2}}-\frac{\sqrt{3}}{4} I_{2,2}^{\mathrm{sub}_{2}}-\frac{3}{4} I_{2,3}^{\mathrm{sub}_{2}} \\
& -\frac{1}{4} I_{3,1}^{\mathrm{sub}_{2}}+\frac{1}{4} I_{3,2}^{\mathrm{sub}_{2}}+\frac{\sqrt{3}}{4} I_{3,3}^{\mathrm{sub}_{2}}-\frac{\sqrt{3}}{4} I_{1,1}^{\mathrm{sub}_{3}}-\frac{\sqrt{3}}{4} I_{1,2}^{\mathrm{sub}_{3}}+\frac{3}{4} I_{1,3}^{\mathrm{sub}_{3}}+\frac{\sqrt{3}}{4} I_{2,1}^{\mathrm{sub}_{3}} \\
& +\frac{\sqrt{3}}{4} I_{2,2}^{\mathrm{sub}_{3}}-\frac{3}{4} I_{2,3}^{\mathrm{sub}_{3}}+\frac{1}{4} I_{3,1}^{\mathrm{sub}_{3}}+\frac{1}{4} I_{3,2}^{\mathrm{sub}_{3}}-\frac{\sqrt{3}}{4} I_{3,3}^{\mathrm{sub}_{3}}, \\
& I_{3,3}=I_{3,3}^{\mathrm{sub}_{1}}+\frac{3}{4} I_{1,1}^{\mathrm{sub}_{2}}+\frac{3}{4} I_{1,2}^{\mathrm{sub}_{2}}-\frac{\sqrt{3}}{4} I_{1,3}^{\mathrm{sub}_{2}}+\frac{3}{4} I_{2,1}^{\mathrm{sub}_{2}}+\frac{3}{4} I_{2,2}^{\mathrm{sub}_{2}}-\frac{\sqrt{3}}{4} I_{2,3}^{\mathrm{sub}_{2}} \\
& -\frac{\sqrt{3}}{4} I_{3,1}^{\mathrm{sub}_{2}}-\frac{\sqrt{3}}{4} I_{3,2}^{\mathrm{sub}_{2}}+\frac{1}{4} I_{3,3}^{\mathrm{sub}_{2}}+\frac{3}{4} I_{1,1}^{\mathrm{sub}_{3}}-\frac{3}{4} I_{1,2}^{\mathrm{sub}_{3}}-\frac{\sqrt{3}}{4} I_{1,3}^{\mathrm{sub}_{3}}-\frac{3}{4} I_{2,1}^{\mathrm{sub}_{3}} \\
& +\frac{3}{4} I_{2,2}^{\mathrm{sub}_{3}}+\frac{\sqrt{3}}{4} I_{2,3}^{\mathrm{sub}_{3}}-\frac{\sqrt{3}}{4} I_{3,1}^{\mathrm{sub}_{3}}+\frac{\sqrt{3}}{4} I_{3,2}^{\mathrm{sub}_{3}}+\frac{1}{4} I_{3,3}^{\mathrm{sub}_{3}} .
\end{aligned}
$$

Finally, the weakly singular integrals (1) and (2) for the case of coincident triangular elements are easily evaluated with the help of the expressions above and the formulas in Equations (7)-(16). 


\section{EDGE ADJACENT INTEGRATION}

\subsection{First analytical integration}

Based upon the coincident integration scheme, we employ here a polar coordinate transformation for the inner integration (triangle Q) to cancel the line of singularity defined by $\xi=\xi^{\prime}=0$ and $\eta=-\eta^{\prime}$,

$$
\eta^{\prime}=\rho \cos (\theta)-\eta, \quad \xi^{\prime}=\rho \sin (\theta) .
$$

The distance function takes the form

$$
R=\sqrt{\beta_{e_{1}} \xi^{2}+\beta_{e_{2}} \xi \rho+\beta_{e_{3}} \rho^{2}},
$$

where

$$
\begin{aligned}
& \beta_{e_{1}}=\left|\boldsymbol{\alpha}_{e_{2}}\right|^{2}, \\
& \beta_{e_{2}}=2\left[\left(\boldsymbol{\alpha}_{e_{1}} \cdot \boldsymbol{\alpha}_{e_{2}}\right) \cos (\theta)+\left(\boldsymbol{\alpha}_{e_{2}} \cdot \boldsymbol{\alpha}_{e_{3}}\right) \sin (\theta)\right], \\
& \beta_{e_{3}}=\left|\boldsymbol{\alpha}_{e_{1}}\right|^{2} \cos ^{2}(\theta)+2\left(\boldsymbol{\alpha}_{e_{1}} \cdot \boldsymbol{\alpha}_{e_{3}}\right) \cos (\theta) \sin (\theta)+\left|\boldsymbol{\alpha}_{e_{3}}\right|^{2} \sin ^{2}(\theta) .
\end{aligned}
$$

and the vectors $\boldsymbol{\alpha}_{e_{1}}, \boldsymbol{\alpha}_{e_{2}}, \boldsymbol{\alpha}_{e_{3}}$ are provided in Equation (20). Again, the integration with respect to $\theta$ should be split into two pieces, as shown in Figure 4(a),

$$
\begin{aligned}
I_{p, q}= & \int_{-1}^{1} \mathrm{~d} \eta \int_{0}^{\xi(\eta)} \psi_{p}(\eta, \xi) \mathrm{d} \xi \\
& \cdot\left[\int_{0}^{\Theta_{1}(\eta)} \mathrm{d} \theta \int_{0}^{L_{1}^{+}} \psi_{q}(\eta, \theta, \rho) \frac{\mathrm{e}^{-\mathrm{j} k R}}{R} \rho \mathrm{d} \rho+\int_{\Theta_{1}(\eta)}^{\pi} \mathrm{d} \theta \int_{0}^{L_{1}^{-}} \psi_{q}(\eta, \theta, \rho) \frac{\mathrm{e}^{-\mathrm{j} k R}}{R} \rho \mathrm{d} \rho\right],
\end{aligned}
$$

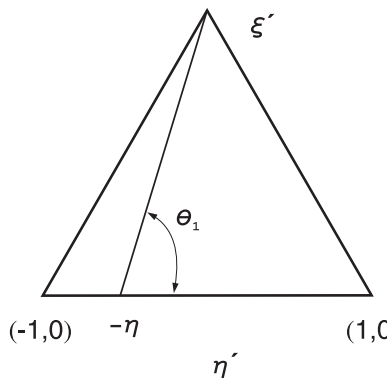

(a)

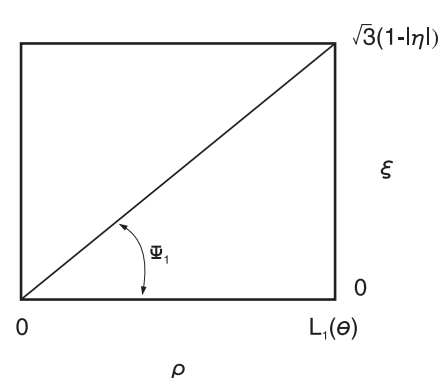

(b)

Figure 4. Polar coordinate transformations employed in the edge adjacent integration: (a) first transformation: $\left\{\eta^{\prime}, \xi^{\prime}\right\} \rightarrow\{\rho, \theta\}$ and (b) second transformation: $\{\rho, \xi\} \rightarrow\{\Lambda, \Psi\}$. 
where

$$
\Theta_{1}(\eta)=\frac{\pi}{2}-\tan ^{-1}\left(\frac{\eta}{\sqrt{3}}\right), \quad L_{1}^{ \pm}=\frac{\sqrt{3}(1 \pm \eta)}{\sin (\theta) \pm \sqrt{3} \cos (\theta)} .
$$

The key feature of the aforementioned transformation is that the break-point in $\theta$ is only a function of $\eta$. Hence, the integration can be rearranged,

$$
\begin{aligned}
I_{p, q}= & \int_{-1}^{1} \mathrm{~d} \eta \int_{0}^{\Theta_{1}(\eta)} \mathrm{d} \theta \int_{0}^{\xi(\eta)} \psi_{p}(\eta, \xi) \mathrm{d} \xi \int_{0}^{L_{1}^{+}} \psi_{q}(\eta, \theta, \rho) \frac{\mathrm{e}^{-\mathrm{j} k R}}{R} \rho \mathrm{d} \rho \\
& +\int_{-1}^{1} \mathrm{~d} \eta \int_{\Theta_{1}(\eta)}^{\pi} \mathrm{d} \theta \int_{0}^{\xi(\eta)} \psi_{p}(\eta, \xi) \mathrm{d} \xi \int_{0}^{L_{1}^{-}} \psi_{q}(\eta, \theta, \rho) \frac{\mathrm{e}^{-\mathrm{j} k R}}{R} \rho \mathrm{d} \rho .
\end{aligned}
$$

As the singularity now occurs when $\rho=\xi=0$, we proceed by introducing a second polar coordinate transformation,

$$
\rho=\Lambda \cos (\Psi), \quad \xi=\Lambda \sin (\Psi),
$$

as shown in Figure 4(b). The distance function in the new parametric system takes the form

$$
R=\Lambda \sqrt{\beta_{e_{1}} \sin ^{2}(\Psi)+\beta_{e_{2}} \sin (\Psi) \cos (\Psi)+\beta_{e_{3}} \cos ^{2}(\Psi)}=\Lambda B(\theta, \Psi)
$$

and the original integral can be written as a sum of 3-D integrals,

$$
I_{p, q}=\sum_{l=0}^{1} \sum_{m=0}^{1} \int_{-1}^{1} \mathrm{~d} \eta \int_{\Theta_{l}}^{\Theta_{l+1}} \mathrm{~d} \theta \int_{\Psi_{m}}^{\Psi_{m+1}} A_{p, q}(\eta, \theta, \Psi) \mathrm{d} \Psi,
$$

since the integral with respect to $\rho$,

$$
A_{p, q}(\eta, \theta, \Psi)=\frac{\cos (\Psi)}{B(\theta, \Psi)} \int_{0}^{L(\eta, \theta)} \psi_{p}(\eta, \Psi, \Lambda) \psi_{q}(\eta, \theta, \Psi, \Lambda) \mathrm{e}^{-\mathrm{j} k B(\theta, \Psi) \Lambda} \Lambda \mathrm{d} \Lambda
$$

can be evaluated analytically. The integration limits in Equation (60) are given by

$$
\Theta_{0}=0, \quad \Theta_{1}=\frac{\pi}{2}-\tan ^{-1}\left(\frac{\eta}{\sqrt{3}}\right), \quad \Theta_{2}=\pi
$$

and

$$
\Psi_{0}=0, \quad \Psi_{1}=\tan ^{-1}\left(\frac{\sqrt{3}(1-|\eta|)}{L(\eta, \theta)}\right), \quad \Psi_{2}=\frac{\pi}{2},
$$

while the integration limits with respect to $\Lambda$ will be shown below. As the angle integrals cannot be evaluated analytically, it will therefore be necessary to rearrange the aforementioned integrals by placing the $\eta$ integral in the innermost position. 


\subsection{Second analytical integration}

As with the coincident integral, the singularity is at $\eta= \pm 1$, and it is convenient to consider $\eta$ positive and negative separately. In the following, we examine the integration over region $\eta \geqslant 0$. Moreover, in the implementation of this method, integration over positive values of $\eta$ suffices, since integration over region $\eta \leqslant 0$ can be easily computed by flipping the elements around and using the $\eta \geqslant 0$ formulas. This is analogues to the rotation procedure employed for the coincident integration.

The additional complication in the edge adjacent case is that, as shown in Figure 4, both the $\theta$ and $\Psi$ integrals are separated into two pieces. In particular, the splitting in $\theta$ is $0<\theta \leqslant \Theta_{1}$ and $\Theta_{1} \leqslant \theta \leqslant \pi$, where $\Theta_{1}$ is defined in Equation (62).

4.2.1. Integration over region $\Theta_{1} \leqslant \theta \leqslant \pi$. In this case, the splitting of $\Psi$ integrals is independent of $\eta$ and, thus, this is the simpler of the two cases. The two integrals are

$$
I_{p, q}^{\theta_{+}}=\int_{0}^{1} \mathrm{~d} \eta \int_{\theta_{\eta}}^{\pi} \mathrm{d} \theta \int_{0}^{\Psi_{1}^{+}} A_{p, q}(\eta, \theta, \Psi) \mathrm{d} \Psi+\int_{0}^{1} \mathrm{~d} \eta \int_{\theta_{\eta}}^{\pi} \mathrm{d} \theta \int_{\Psi_{1}^{+}}^{\pi / 2} A_{p, q}(\eta, \theta, \Psi) \mathrm{d} \Psi,
$$

where the upper limit on $\Lambda$ is different in the two terms,

$$
\Lambda_{L}= \begin{cases}\frac{L_{1}^{-}}{\cos (\Psi)}, & 0<\Psi<\Psi_{1}, \\ \frac{\xi(\eta)}{\sin (\Psi)}, & \Psi_{1}<\Psi<\pi / 2 .\end{cases}
$$

Moreover,

$$
\theta_{\eta} \equiv \Theta_{1}=\frac{\pi}{2}-\tan ^{-1}\left(\frac{\eta}{\sqrt{3}}\right)
$$

and the integral with respect to $\Psi$ is split at

$$
\Psi_{1}^{+}=\tan ^{-1}(\sin (\theta)-\sqrt{3} \cos (\theta))
$$

The important simplification in this case comes from the fact that $\Psi_{1}$ is not a function of $\eta$. Hence, once $\eta$ and $\theta$ are interchanged, the $\eta$ can be moved immediately past the $\Psi$ integral.

Noting that $\theta_{\eta}(0)=\pi / 2$ and $\theta_{\eta}(1)=\pi / 3$, the geometry for interchanging $\eta$ and $\theta$ is shown in Figure 5(a). Inverting the relationship between $\eta$ and $\theta$ yields

$$
\eta_{\theta}=\frac{\sqrt{3}}{\tan (\theta)}
$$

while switching the integrals results in

$$
\begin{aligned}
I_{p, q}^{\theta_{+}}= & \int_{\pi / 2}^{\pi} \mathrm{d} \theta \int_{0}^{\Psi_{1}^{+}} \mathrm{d} \Psi \int_{0}^{1} A_{p, q}(\eta, \theta, \Psi) \mathrm{d} \eta+\int_{\pi / 3}^{\pi / 2} \mathrm{~d} \theta \int_{0}^{\Psi_{1}^{+}} \mathrm{d} \Psi \int_{\eta_{\theta}}^{1} A_{p, q}(\eta, \theta, \Psi) \mathrm{d} \eta \\
& +\int_{\pi / 2}^{\pi} \mathrm{d} \theta \int_{\Psi_{1}^{+}}^{\pi / 2} \mathrm{~d} \Psi \int_{0}^{1} A_{p, q}(\eta, \theta, \Psi) \mathrm{d} \eta+\int_{\pi / 3}^{\pi / 2} \mathrm{~d} \theta \int_{\Psi_{1}^{+}}^{\pi / 2} \mathrm{~d} \Psi \int_{\eta_{\theta}}^{1} A_{p, q}(\eta, \theta, \Psi) \mathrm{d} \eta
\end{aligned}
$$



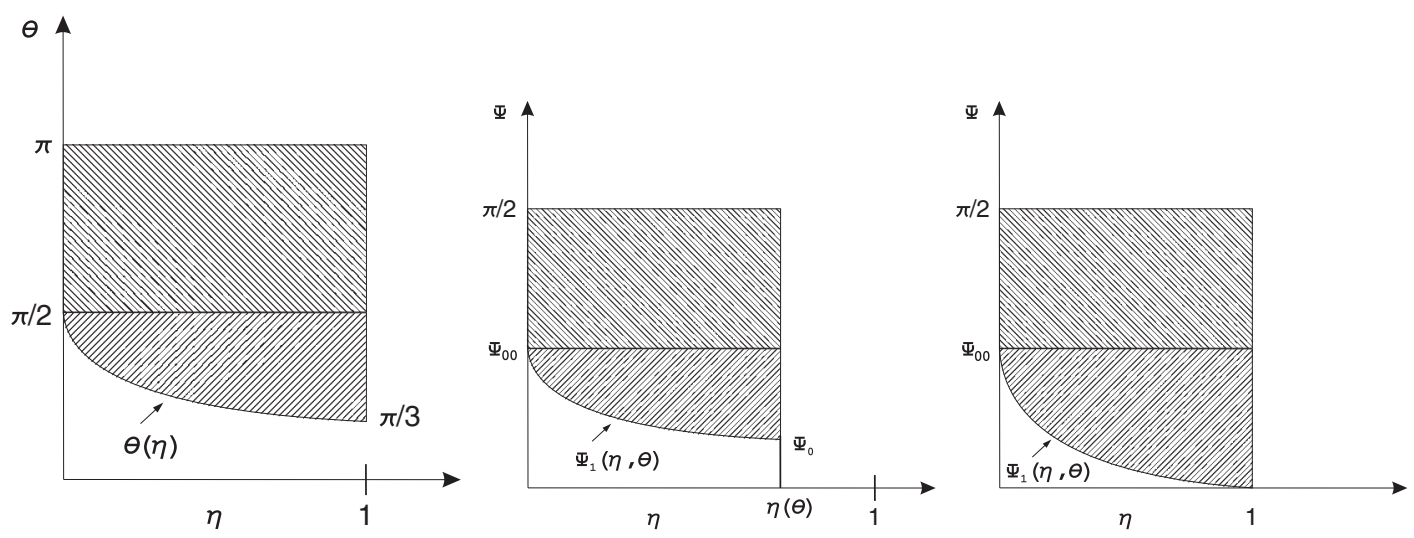

(b)

(c)

Figure 5. Polar coordinate transformations employed in the edge adjacent integration: (a) the $\{\eta, \theta\}$ domain for the first shift of the integral, $\Theta_{1} \leqslant \theta \leqslant \pi$; (b) the domain for interchanging the integrals $\{\eta$, $\Psi\}$, for a fixed value of $\theta$ and $\eta(\theta)<1 \quad\left(0<\theta \leqslant \Theta_{1}\right)$; and (c) the domain for interchanging the integrals $\{\eta, \Psi\}$, for a fixed value of $\theta$ and $\eta(\theta)=1\left(0<\theta \leqslant \Theta_{1}\right)$.

As before, the integration with respect to $\eta$ can be carried out analytically, leading to the following expressions:

$$
\begin{aligned}
I_{p, q}^{\theta_{+}}= & \int_{\pi / 2}^{\pi} \mathrm{d} \theta \int_{0}^{\Psi_{1}^{+}} X_{p, q}^{a}(\theta, \Psi) \mathrm{d} \Psi+\int_{\pi / 3}^{\pi / 2} \mathrm{~d} \theta \int_{0}^{\Psi_{1}^{+}} X_{p, q}^{b}(\theta, \Psi) \mathrm{d} \Psi \\
& +\int_{\pi / 2}^{\pi} \mathrm{d} \theta \int_{\Psi_{1}^{+}}^{\pi / 2} X_{p, q}^{c}(\theta, \Psi) \mathrm{d} \Psi+\int_{\pi / 3}^{\pi / 2} \mathrm{~d} \theta \int_{\Psi_{1}^{+}}^{\pi / 2} X_{p, q}^{d}(\theta, \Psi) \mathrm{d} \Psi .
\end{aligned}
$$

4.2.2. Integration over region $0<\theta \leqslant \Theta_{1}$. In this case, the breakpoint in $\Psi$ is a function of $\eta$ and shifting things around will now produce seven integrals instead of the aforementioned four. The two starting integrals are given by

$$
I_{p, q}^{\theta_{-}}=\int_{0}^{1} \mathrm{~d} \eta \int_{0}^{\theta_{\eta}} \mathrm{d} \theta \int_{0}^{\Psi_{1}^{-}} A_{p, q}(\eta, \theta, \Psi) \mathrm{d} \Psi+\int_{0}^{1} \mathrm{~d} \eta \int_{0}^{\theta_{\eta}} \mathrm{d} \theta \int_{\Psi_{1}^{-}}^{\pi / 2} A_{p, q}(\eta, \theta, \Psi) \mathrm{d} \Psi,
$$

where, again, $\Lambda_{L}$ is given by Equation (65) with $L_{1}^{-}$, but now

$$
\Psi_{1}^{-}=\tan ^{-1}\left(\frac{\xi(\eta)}{L_{1}^{+}}\right)=\tan ^{-1}\left(\frac{1-\eta}{1+\eta}[\sin (\theta)+\sqrt{3} \cos (\theta)]\right) .
$$

As in the previous section, the $\theta$ and $\eta$ integrals are easily interchanged, the domain being the region below the $\theta(\eta)$ curve in Figure 5(a). This results in the four integrals

$$
\begin{aligned}
I_{p, q}^{\theta_{-}}= & \int_{0}^{\pi / 3} \mathrm{~d} \theta \int_{0}^{1} \mathrm{~d} \eta \int_{0}^{\Psi_{1}^{-}} A_{p, q}(\eta, \theta, \Psi) \mathrm{d} \Psi+\int_{\pi / 3}^{\pi / 2} \mathrm{~d} \theta \int_{0}^{\eta_{\theta}} \mathrm{d} \eta \int_{0}^{\Psi_{1}^{-}} A_{p, q}(\eta, \theta, \Psi) \mathrm{d} \Psi \\
& +\int_{0}^{\pi / 3} \mathrm{~d} \theta \int_{0}^{1} \mathrm{~d} \eta \int_{\Psi_{1}^{-}}^{\pi / 2} A_{p, q}(\eta, \theta, \Psi) \mathrm{d} \Psi+\int_{\pi / 3}^{\pi / 2} \mathrm{~d} \theta \int_{0}^{\eta_{\theta}} \mathrm{d} \eta \int_{\Psi_{1}^{-}}^{\pi / 2} A_{p, q}(\eta, \theta, \Psi) \mathrm{d} \Psi,
\end{aligned}
$$


where $\eta_{\theta}$ is given in (68). Using the fact that $\Psi_{1}(\eta=1, \theta)=0$, the geometry for interchanging $\eta$ and $\Psi$ is shown in Figure 5(b) $(\eta(\theta)<1)$ and in Figure 5(c), the region below the curve for $\eta(\theta)=1$.

Integration over region $0<\Psi \leqslant \Psi_{1}^{-}$: Moving the $\eta$ integral to the front in the first two integrals in Equation (73) $\left(0<\Psi<\Psi_{1}^{-}\right)$results in

$$
\begin{aligned}
I_{p, q}^{\theta_{-}, \Psi_{-}}= & \int_{0}^{\pi / 3} \mathrm{~d} \theta \int_{0}^{\Psi_{00}} \mathrm{~d} \Psi \int_{0}^{\Psi_{\eta}} A_{p, q}(\eta, \theta, \Psi) \mathrm{d} \eta+\int_{\pi / 3}^{\pi / 2} \mathrm{~d} \theta \int_{0}^{\Psi_{\theta}} \mathrm{d} \Psi \int_{0}^{\eta_{\theta}} A_{p, q}(\eta, \theta, \Psi) \mathrm{d} \eta \\
& +\int_{\pi / 3}^{\pi / 2} \mathrm{~d} \theta \int_{\Psi_{\theta}}^{\Psi_{00}} \mathrm{~d} \Psi \int_{0}^{\Psi_{\eta}} A_{p, q}(\eta, \theta, \Psi) \mathrm{d} \eta
\end{aligned}
$$

where

$$
\begin{aligned}
& \Psi_{00}=\Psi_{1}^{+}(\eta=0)=\tan ^{-1}(\sin (\theta)+\sqrt{3} \cos (\theta)), \\
& \Psi_{\theta}=\Psi_{1}^{+}\left(\eta=\eta_{\theta}\right)=\tan ^{-1}\left(\frac{1-\eta_{\theta}}{1+\eta_{\theta}}[\sin (\theta)+\sqrt{3} \cos (\theta)]\right)
\end{aligned}
$$

and

$$
\Psi_{\eta}=\frac{1-\frac{\tan (\Psi)}{\sin (\theta)+\sqrt{3} \cos (\theta)}}{1+\frac{\tan (\Psi)}{\sin (\theta)+\sqrt{3} \cos (\theta)}}=\frac{1-\gamma}{1+\gamma} .
$$

The expressions after the analytical integration with respect to $\eta$ are given by:

$$
\begin{aligned}
I_{p, q}^{\theta_{-}, \Psi_{-}}= & \int_{0}^{\pi / 3} \mathrm{~d} \theta \int_{0}^{\Psi_{00}} X_{p, q}^{e}(\theta, \Psi) \mathrm{d} \Psi+\int_{\pi / 3}^{\pi / 2} \mathrm{~d} \theta \int_{0}^{\Psi_{\theta}} X_{p, q}^{f}(\theta, \Psi) \mathrm{d} \Psi \\
& +\int_{\pi / 3}^{\pi / 2} \mathrm{~d} \theta \int_{\Psi_{\theta}}^{\Psi_{00}} X_{p, q}^{g}(\theta, \Psi) \mathrm{d} \Psi
\end{aligned}
$$

Integration over region $\Psi_{1}^{-}<\Psi \leqslant \pi / 2$ : Similarly, the second two integrals in Equation (73) $\left(\Psi_{1}^{-}<\Psi<\pi / 2\right)$ become

$$
\begin{aligned}
I_{p, q}^{\theta_{-}, \Psi_{+}}= & \int_{0}^{\pi / 3} \mathrm{~d} \theta \int_{0}^{\Psi_{00}} \mathrm{~d} \Psi \int_{\Psi_{\eta}}^{1} A_{p, q}(\eta, \theta, \Psi) \mathrm{d} \eta+\int_{0}^{\pi / 3} \mathrm{~d} \theta \int_{\Psi_{00}}^{\pi / 2} \mathrm{~d} \Psi \int_{0}^{1} A_{p, q}(\eta, \theta, \Psi) \mathrm{d} \eta \\
& +\int_{\pi / 3}^{\pi / 2} \mathrm{~d} \theta \int_{\Psi_{\theta}}^{\Psi_{00}} \mathrm{~d} \Psi \int_{\Psi_{\eta}}^{\eta_{\theta}} A_{p, q}(\eta, \theta, \Psi) \mathrm{d} \eta \\
& +\int_{\pi / 3}^{\pi / 2} \mathrm{~d} \theta \int_{\Psi_{00}}^{\pi / 2} \mathrm{~d} \Psi \int_{0}^{\eta_{\theta}} A_{p, q}(\eta, \theta, \Psi) \mathrm{d} \eta,
\end{aligned}
$$


resulting, after the analytical integration with respect to $\eta$, in the following expressions:

$$
\begin{aligned}
I_{p, q}^{\theta_{-}, \Psi_{+}}= & \int_{0}^{\pi / 3} \mathrm{~d} \theta \int_{0}^{\Psi_{00}} X_{p, q}^{h}(\theta, \Psi) \mathrm{d} \Psi+\int_{0}^{\pi / 3} \mathrm{~d} \theta \int_{\Psi_{00}}^{\pi / 2} X_{p, q}^{i}(\theta, \Psi) \mathrm{d} \Psi \\
& +\int_{\pi / 3}^{\pi / 2} \mathrm{~d} \theta \int_{\Psi_{\theta}}^{\Psi_{00}} X_{p, q}^{k}(\theta, \Psi) \mathrm{d} \Psi+\int_{\pi / 3}^{\pi / 2} \mathrm{~d} \theta \int_{\Psi_{00}}^{\pi / 2} X_{p, q}^{l}(\theta, \Psi) \mathrm{d} \Psi
\end{aligned}
$$

Finally, by adding all the components from Equations (70), (78) and (80), we end up to the following formula for the integration over positive values of $\eta$ :

$$
I_{p, q}^{\eta_{+}}=I_{p, q}^{\theta_{-}}+I_{p, q}^{\theta_{+}}=I_{p, q}^{\theta_{-}, \Psi_{-}}+I_{p, q}^{\theta_{-}, \Psi_{+}}+I_{p, q}^{\theta_{+}} .
$$

More specifically, the weakly singular integrals for $\eta>0$ have been reduced to the following 2-D smooth integrals:

$$
\begin{aligned}
I_{p, q}^{\eta_{+}}= & \int_{\pi / 2}^{\pi} \mathrm{d} \theta \int_{0}^{\Psi_{1}^{+}} X_{p, q}^{a}(\theta, \Psi) \mathrm{d} \Psi+\int_{\pi / 3}^{\pi / 2} \mathrm{~d} \theta \int_{0}^{\Psi_{1}^{+}} X_{p, q}^{b}(\theta, \Psi) \mathrm{d} \Psi+\int_{\pi / 2}^{\pi} \mathrm{d} \theta \int_{\Psi_{1}^{+}}^{\pi / 2} X_{p, q}^{c}(\theta, \Psi) \mathrm{d} \Psi \\
& +\int_{\pi / 3}^{\pi / 2} \mathrm{~d} \theta \int_{\Psi_{1}^{+}}^{\pi / 2} X_{p, q}^{d}(\theta, \Psi) \mathrm{d} \Psi+\int_{0}^{\pi / 3} \mathrm{~d} \theta \int_{0}^{\Psi_{00}} X_{p, q}^{e}(\theta, \Psi) \mathrm{d} \Psi \\
& +\int_{\pi / 3}^{\pi / 2} \mathrm{~d} \theta \int_{0}^{\Psi_{\theta}} X_{p, q}^{f}(\theta, \Psi) \mathrm{d} \Psi+\int_{\pi / 3}^{\pi / 2} \mathrm{~d} \theta \int_{\Psi_{\theta}}^{\Psi_{00}} X_{p, q}^{g}(\theta, \Psi) \mathrm{d} \Psi \\
& +\int_{0}^{\pi / 3} \mathrm{~d} \theta \int_{0}^{\Psi_{00}} X_{p, q}^{h}(\theta, \Psi) \mathrm{d} \Psi+\int_{0}^{\pi / 3} \mathrm{~d} \theta \int_{\Psi_{00}}^{\pi / 2} X_{p, q}^{i}(\theta, \Psi) \mathrm{d} \Psi \\
& +\int_{\pi / 3}^{\pi / 2} \mathrm{~d} \theta \int_{\Psi_{\theta}}^{\Psi_{00}} X_{p, q}^{k}(\theta, \Psi) \mathrm{d} \Psi+\int_{\pi / 3}^{\pi / 2} \mathrm{~d} \theta \int_{\Psi_{00}}^{\pi / 2} X_{p, q}^{l}(\theta, \Psi) \mathrm{d} \Psi,
\end{aligned}
$$

where the integrands are analytically evaluated smooth functions. It is worth mentioning in this point that the dimensionality reduction of the original 4-D integrals in both the edge adjacent case and vertex adjacent case (as will be shown next) is limited compared with the coincident case due to the algebraic complexity of the distance function after the variable transformations. To be more precise, the dimensionality of the final integrals is equal to the independent variables in the expressions $\alpha(\theta), B(\theta, \Psi)$ and $\Gamma\left(\theta_{p}, \theta_{q}, \Psi\right)$ for, respectively, the coincident, edge adjacent and vertex adjacent case.

\subsection{Final formulas}

Similar to the coincident integration, the remaining case (integration over negative values of $\eta$ ) can be handled by simply flipping the master triangles (triangles in the equilateral triangle parametric 
space) and employing the formulas for the $\eta>0$ case. More specifically, the expressions for the $\eta<0$ case are given by the following formula:

$$
I_{p, q}^{\eta_{-}}=I_{p, q}^{\eta_{+}} \mid \begin{aligned}
& \beta_{e_{1} \rightarrow \beta_{e_{1,2}}}, \\
& \beta_{e_{2} \rightarrow \beta_{e_{2,2}}} \\
& \beta_{e_{3} \rightarrow \beta_{e_{3,2}}}
\end{aligned},
$$

where

$$
\begin{aligned}
& \beta_{e_{1,2}}=\left|\boldsymbol{\alpha}_{e_{2}}\right|^{2}, \\
& \beta_{e_{2,2}}=2\left[\left(\boldsymbol{\alpha}_{e_{2}} \cdot \boldsymbol{\alpha}_{e_{3}}\right) \sin (\theta)-\left(\boldsymbol{\alpha}_{e_{1}} \cdot \boldsymbol{\alpha}_{e_{2}}\right) \cos (\theta)\right], \\
& \beta_{e_{3,2}}=\left|\boldsymbol{\alpha}_{e_{1}}\right|^{2} \cos ^{2}(\theta)-2\left(\boldsymbol{\alpha}_{e_{1}} \cdot \boldsymbol{\alpha}_{e_{3}}\right) \cos (\theta) \sin (\theta)+\left|\boldsymbol{\alpha}_{e_{3}}\right|^{2} \sin ^{2}(\theta) .
\end{aligned}
$$

Finally, the weakly singular integrals $I_{p, q}$ can be evaluated as a function of the subintegrals $I_{p, q}^{\eta_{+}}$and $I_{p, q}^{\eta_{-}}$,

$$
\begin{array}{llll}
I_{1,1}=I_{1,1}^{\eta_{+}}+I_{1,1}^{\eta_{-}}, & I_{1,2}=I_{1,2}^{\eta_{+}}-I_{1,2}^{\eta_{-}}, & I_{1,3}=I_{1,3}^{\eta_{+}}+I_{1,3}^{\eta_{-}}, & I_{2,1}=I_{2,1}^{\eta_{+}}-I_{2,1}^{\eta_{-}}, \\
I_{2,2}=I_{2,2}^{\eta_{+}}+I_{2,2}^{\eta_{-}}, & I_{2,3}=I_{2,3}^{\eta_{+}}-I_{2,3}^{\eta_{-}}, & I_{3,1}=I_{3,1}^{\eta_{+}}+I_{3,1}^{\eta_{-}}, & I_{3,2}=I_{3,2}^{\eta_{+}}-I_{3,2}^{\eta_{-}}, \\
I_{3,3}=I_{3,3}^{\eta_{+}}+I_{3,3}^{\eta_{-}} . &
\end{array}
$$

\section{VERTEX ADJACENT INTEGRATION}

As discussed in Reference [28], the vertex adjacent integrals are separately finite, the singularity being limited to a single point in the 4-D integration. Thus, there is a variety of methods that can be used to evaluate these terms. Here, we keep the baseline presented in the previous sections, mainly for the sake of homogeneity. To begin, we orient the elements so that the singular point is $\eta=-1$ and $\eta^{\prime}=-1$. Next, we introduce the following separate polar coordinate systems for each element (Figure 6(a)):

$$
\begin{aligned}
\eta^{\prime} & =\rho_{q} \cos \left(\theta_{q}\right)-1, \quad \xi^{\prime}=\rho_{q} \sin \left(\theta_{q}\right), \\
\eta & =\rho_{p} \cos \left(\theta_{p}\right)-1, \quad \xi=\rho_{p} \sin \left(\theta_{p}\right) .
\end{aligned}
$$

Consequently, the distance function takes the form

$$
R=\sqrt{\beta_{v_{1}} \rho_{p}^{2}+\beta_{v_{2}} \rho_{p} \rho_{q}+\beta_{v_{3}} \rho_{q}^{2}}
$$

where

$$
\begin{aligned}
& \beta_{v_{1}}=\left|\boldsymbol{\alpha}_{v_{1}} \cos \left(\theta_{p}\right)+\boldsymbol{\alpha}_{v_{2}} \sin \left(\theta_{p}\right)\right|^{2}, \\
& \beta_{v_{2}}=-2\left[\left(\boldsymbol{\alpha}_{v_{1}} \cos \left(\theta_{p}\right)+\boldsymbol{\alpha}_{v_{2}} \sin \left(\theta_{p}\right)\right) \cdot\left(\boldsymbol{\alpha}_{v_{3}} \cos \left(\theta_{q}\right)-\boldsymbol{\alpha}_{v_{4}} \sin \left(\theta_{q}\right)\right)\right], \\
& \beta_{v_{3}}=\left|\boldsymbol{\alpha}_{v_{3}} \cos \left(\theta_{q}\right)-\boldsymbol{\alpha}_{v_{4}} \sin \left(\theta_{q}\right)\right|^{2} .
\end{aligned}
$$




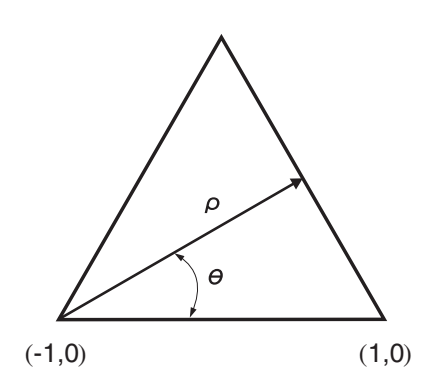

(a)

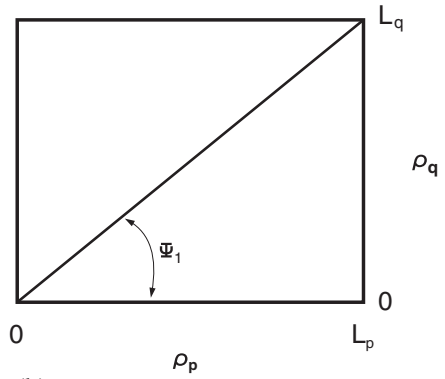

(b)

Figure 6. Polar coordinate transformations employed in the vertex adjacent integration: (a) first transformation: $\{\eta, \xi\} \rightarrow\left\{\rho_{p}, \theta_{p}\right\}$ and $\left\{\eta^{\prime}, \xi^{\prime}\right\} \rightarrow\left\{\rho_{q}, \theta_{q}\right\}$ and (b) second transformation: $\left\{\rho_{p}, \rho_{q}\right\} \rightarrow\{\Lambda, \Psi\}$.

This results in an integral of the form

$$
\begin{aligned}
I_{p, q} & =\int_{0}^{\pi / 3} \mathrm{~d} \theta_{p} \int_{0}^{L_{p}\left(\theta_{p}\right)} \psi_{p}\left(\rho_{p}, \theta_{p}\right) \rho_{p} \mathrm{~d} \rho_{p} \int_{0}^{\pi / 3} \mathrm{~d} \theta_{q} \int_{0}^{L_{q}\left(\theta_{q}\right)} \psi_{q}\left(\rho_{q}, \theta_{q}\right) \frac{\mathrm{e}^{-\mathrm{j} k R}}{R} \rho_{q} \mathrm{~d} \rho_{q} \\
& =\int_{0}^{\pi / 3} \mathrm{~d} \theta_{p} \int_{0}^{\pi / 3} \mathrm{~d} \theta_{q} \int_{0}^{L_{p}\left(\theta_{p}\right)} \psi_{p}\left(\rho_{p}, \theta_{p}\right) \rho_{p} \mathrm{~d} \rho_{p} \int_{0}^{L_{q}\left(\theta_{q}\right)} \psi_{q}\left(\rho_{q}, \theta_{q}\right) \frac{\mathrm{e}^{-\mathrm{j} k R}}{R} \rho_{q} \mathrm{~d} \rho_{q},
\end{aligned}
$$

where

$$
L_{p}\left(\theta_{p}\right)=\frac{2 \sqrt{3}}{\sin \left(\theta_{p}\right)+\sqrt{3} \cos \left(\theta_{p}\right)}, \quad L_{q}\left(\theta_{q}\right)=\frac{2 \sqrt{3}}{\sin \left(\theta_{q}\right)+\sqrt{3} \cos \left(\theta_{q}\right)} .
$$

The singularity is at the common vertex $\rho_{p}=\rho_{q}=0$, justifying the use of one further polar coordinate transformation,

$$
\rho_{p}=\Lambda \cos (\Psi), \quad \rho_{q}=\Lambda \sin (\Psi) .
$$

After the new transformation, the distance function takes the form

$$
R=\Lambda \sqrt{\beta_{v_{1}} \cos ^{2}(\Psi)+\beta_{v_{2}} \sin (\Psi) \cos (\Psi)+\beta_{v_{3}} \sin ^{2}(\Psi)}=\Lambda \Gamma\left(\theta_{p}, \theta_{q}, \Psi\right) .
$$

As indicated in Figure 6(b), the $\left\{\rho_{p}, \rho_{q}\right\}$ domain is a rectangle and the $\Psi$ integration must be taken in two pieces, as follows:

$$
\begin{aligned}
I_{p, q}= & \int_{0}^{\pi / 3} \mathrm{~d} \theta_{p} \int_{0}^{\pi / 3} \mathrm{~d} \theta_{q} \\
& \times\left[\int_{0}^{\Psi_{1}} \cos (\Psi) \sin (\Psi) \psi_{p}\left(\theta_{p}, \Psi, \Lambda\right) \mathrm{d} \Psi \int_{0}^{L_{1}(\Psi)} \psi_{q}\left(\theta_{q}, \Psi, \Lambda\right) \frac{\mathrm{e}^{-\mathrm{j} k \Lambda \Gamma\left(\theta_{p}, \theta_{q}, \Psi\right)}}{\Gamma\left(\theta_{p}, \theta_{q}, \Psi\right)} \Lambda^{2} \mathrm{~d} \Lambda\right.
\end{aligned}
$$




$$
\begin{aligned}
& +\int_{\Psi_{1}}^{\pi / 2} \cos (\Psi) \sin (\Psi) \psi_{p}\left(\theta_{p}, \Psi, \Lambda\right) \mathrm{d} \Psi \\
& \left.\times \int_{0}^{L_{2}(\Psi)} \psi_{q}\left(\theta_{q}, \Psi, \Lambda\right) \frac{\mathrm{e}^{-\mathrm{j} k \Lambda \Gamma\left(\theta_{p}, \theta_{q}, \Psi\right)}}{\Gamma\left(\theta_{p}, \theta_{q}, \Psi\right)} \Lambda^{2} d \Lambda\right]
\end{aligned}
$$

where

$$
L_{1}(\Psi)=\frac{L_{p}\left(\theta_{p}\right)}{\cos (\Psi)}, \quad L_{2}(\Psi)=\frac{L_{q}\left(\theta_{q}\right)}{\sin (\psi)}
$$

and

$$
\Psi_{1}=\tan ^{-1}\left(\frac{L_{q}\left(\theta_{q}\right)}{L_{p}\left(\theta_{p}\right)}\right)
$$

Finally, the weakly singular integrals in Equation (5) are given by

$$
I_{p, q}=\int_{0}^{\pi / 3} \mathrm{~d} \theta_{p} \int_{0}^{\pi / 3} \mathrm{~d} \theta_{q}\left[\int_{0}^{\Psi_{1}} \Omega_{p, q}\left(L_{1}\right) \mathrm{d} \Psi+\int_{\Psi_{1}}^{\pi / 2} \Omega_{p, q}\left(L_{2}\right) \mathrm{d} \Psi\right],
$$

where

$$
\Omega_{p, q}\left(L_{m}\right)=\int_{0}^{L_{m}} \cos (\Psi) \sin (\Psi) \psi_{p}\left(\theta_{p}, \Psi, \Lambda\right) \psi_{q}\left(\theta_{q}, \Psi, \Lambda\right) \frac{\mathrm{e}^{-\mathrm{j} k \Lambda \Gamma\left(\theta_{p}, \theta_{q}, \Psi\right)}}{\Gamma\left(\theta_{p}, \theta_{q}, \Psi\right)} \Lambda^{2} \mathrm{~d} \Lambda
$$

are analytically evaluated smooth functions.

\section{NUMERICAL RESULTS}

In this section, we will present some numerical results for the comparison of the direct evaluation method with all the methods that are mostly used for tackling the problem of the weakly singular integrals evaluation in Galerkin mixed potential integral equation formulations.

\subsection{Coincident integration}

More specifically, in the coincident integration case the proposed scheme will be compared with both the singularity subtraction method (utilizing the analytical formulas presented in Reference [7]) and the singularity cancellation method (as described in Reference [19]). As a typical weakly singular integral test problem, we have selected the scalar potential integral over a unit triangle with $k=1$. The reference solution of the integral (absolute error less than $10^{-16}$ ) is directly copied from Reference [26] as $I_{\text {ref }}=0.952716973790348-\mathrm{j} 0.240945897671652$.

In Figure 7, the relative error in calculating the real part (singular portion) and the imaginary part (nonsingular portion) of the weakly singular integral (2) using the three methods is shown. As it is obvious, the reduction of the dimensionality of the original 4-D integral into a 1-D numerical integration via a completely analytical scheme, has resulted in formulas which provide high accuracy with far less computational effort. In this point, it is worth mentioning that throughout 

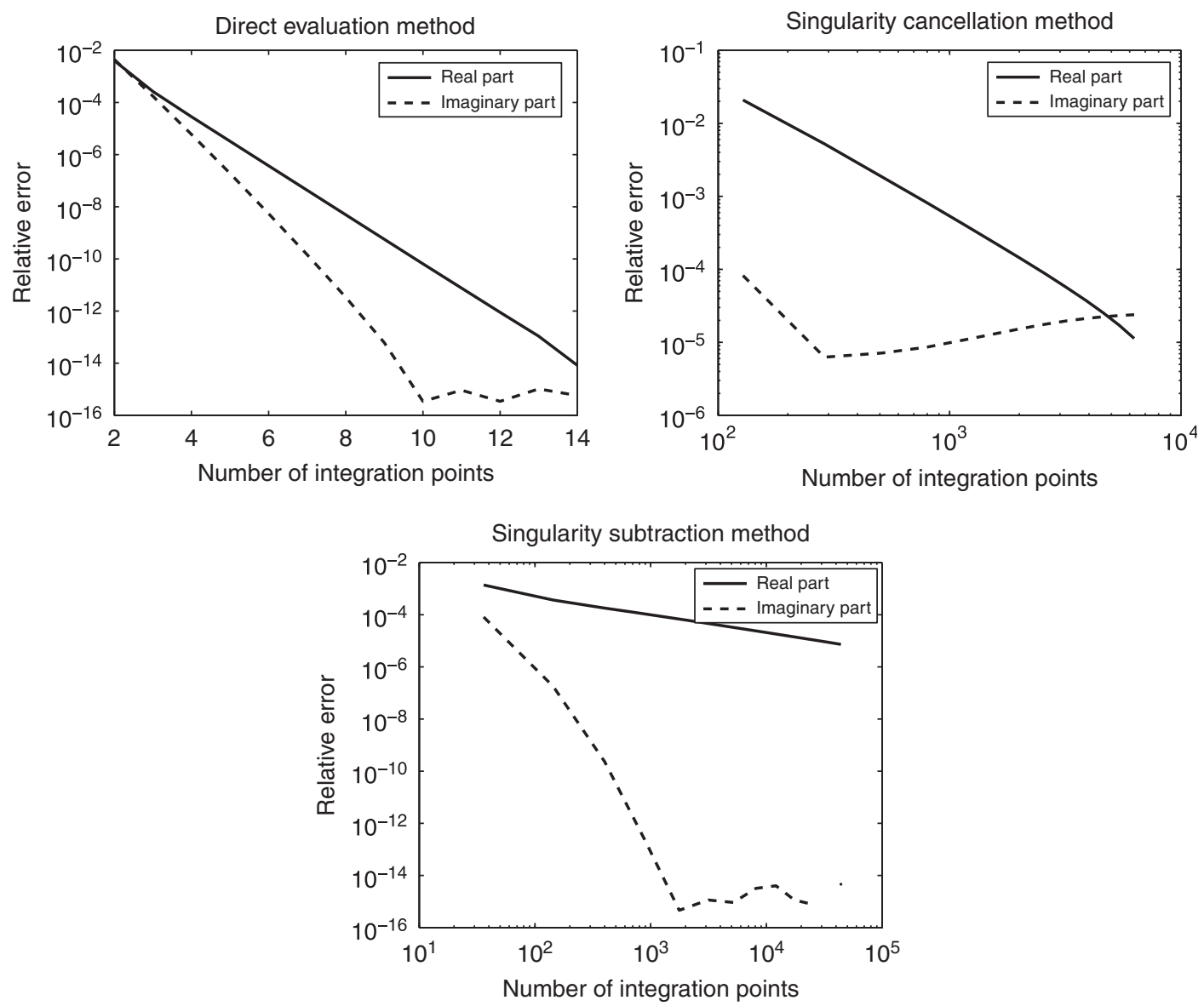

Figure 7. Relative error in calculating the real and the imaginary part of the weakly singular integral $I_{s}^{\text {sing }}$ over the unit triangle.

the whole section the comparison is performed in terms of the actual number of integration points of the associated cubature and, also, that all the multidimensional integrals are computed via products of Gauss quadrature rules. Of course, we have to take into account also the small overhead in the direct evaluation scheme arising from the multiple 1-D integrations, as shown in Equation (46).

Next, a more general example, besides the aforementioned benchmark problem, is presented for the case of a linear basis function. More specifically, in Figure 8, the relative error in calculating the weakly singular integral $I_{1,1}^{\text {sing }}$ for a triangle of worse quality factor $\left(\boldsymbol{r}_{1}=(0,0,0), \boldsymbol{r}_{2}=(1,0,0)\right.$, $\left.\boldsymbol{r}_{3}=\left(\frac{1}{3}, \frac{1}{6}, 0\right)\right)$ and a linear basis function with $k=1$ is shown. As expected, all three methods comprise behavior similar to that of the benchmark problem, only this time more integration points are needed. It is important to mention that for the last example, as well as for the next examples that shall be presented herein, the converged solution of the direct evaluation scheme is taken as a reference. 

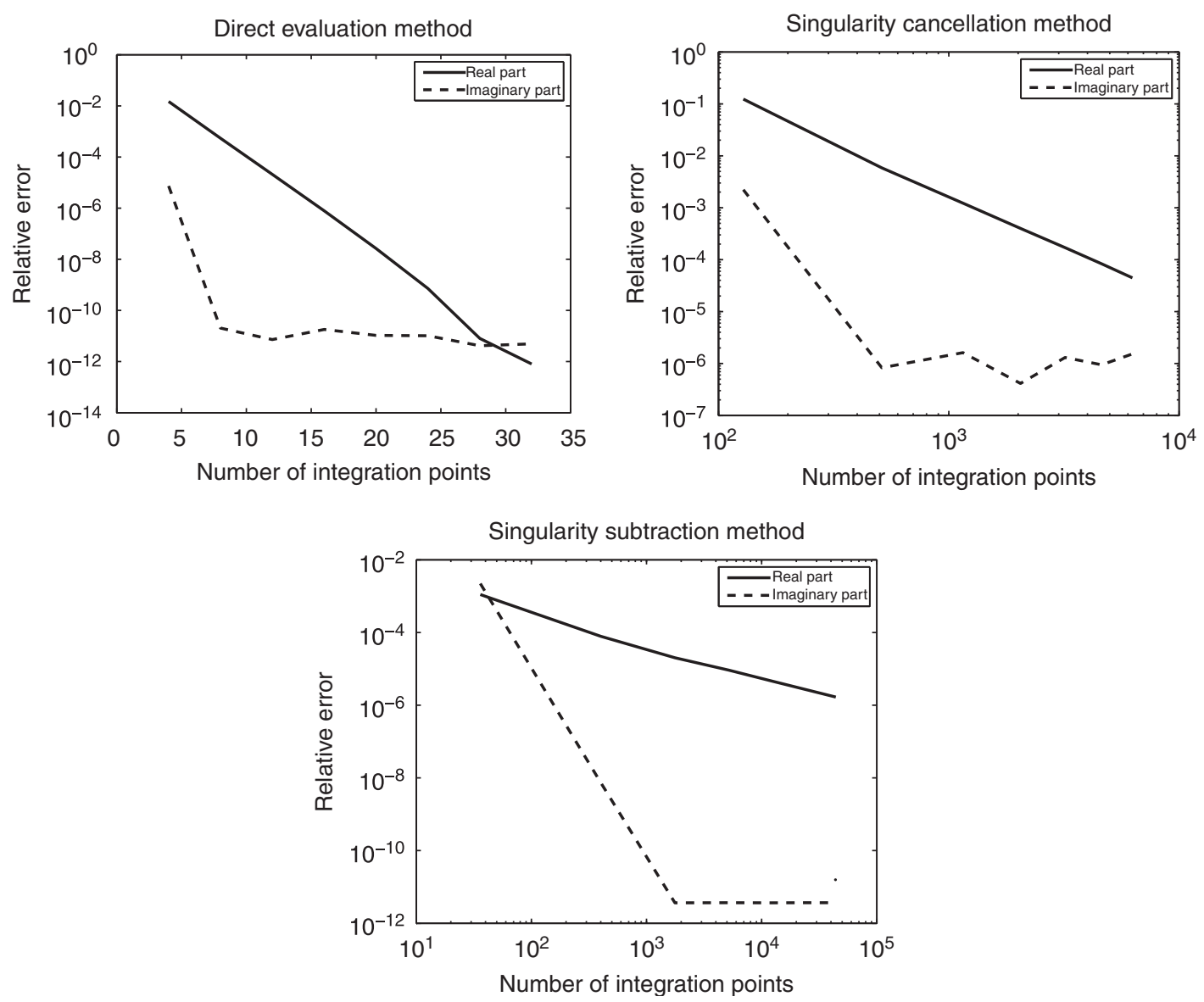

Figure 8 . Relative error in calculating the real and the imaginary part of the weakly singular integral $I_{1,1}^{\text {sing }}$ over a triangle with the following vertices: $\boldsymbol{r}_{1}=(0,0,0), \boldsymbol{r}_{2}=(1,0,0), \boldsymbol{r}_{3}=\left(\frac{1}{3}, \frac{1}{6}, 0\right)$.

\subsection{Edge adjacent integration}

In the edge adjacent case, we slightly modify the comparison procedure. More specifically, the direct evaluation method is compared with the fully numerical method often used to compute the integrals with an isolated singularity along the shared edge. In addition, we have included in the first example the results obtained by utilizing only the first analytical integration in the direct evaluation method. This is almost equivalent to the singularity cancellation concept, since the Jacobian of the first transformation is enough in order to cancel out the singularity of the distance function in the denominator of the free-space Green's function. Consequently, in Figure 9 the relative error in computing the weakly singular integral (2) with $k=1$ over the edge adjacent triangles defined by the vertices: $\boldsymbol{r}_{1}=(1,1,0), \boldsymbol{r}_{2}=(2,1,0), \boldsymbol{r}_{3}=(1,2,0), \boldsymbol{r}_{4}=(1,0,0)$ using the aforementioned three methods, is shown. 

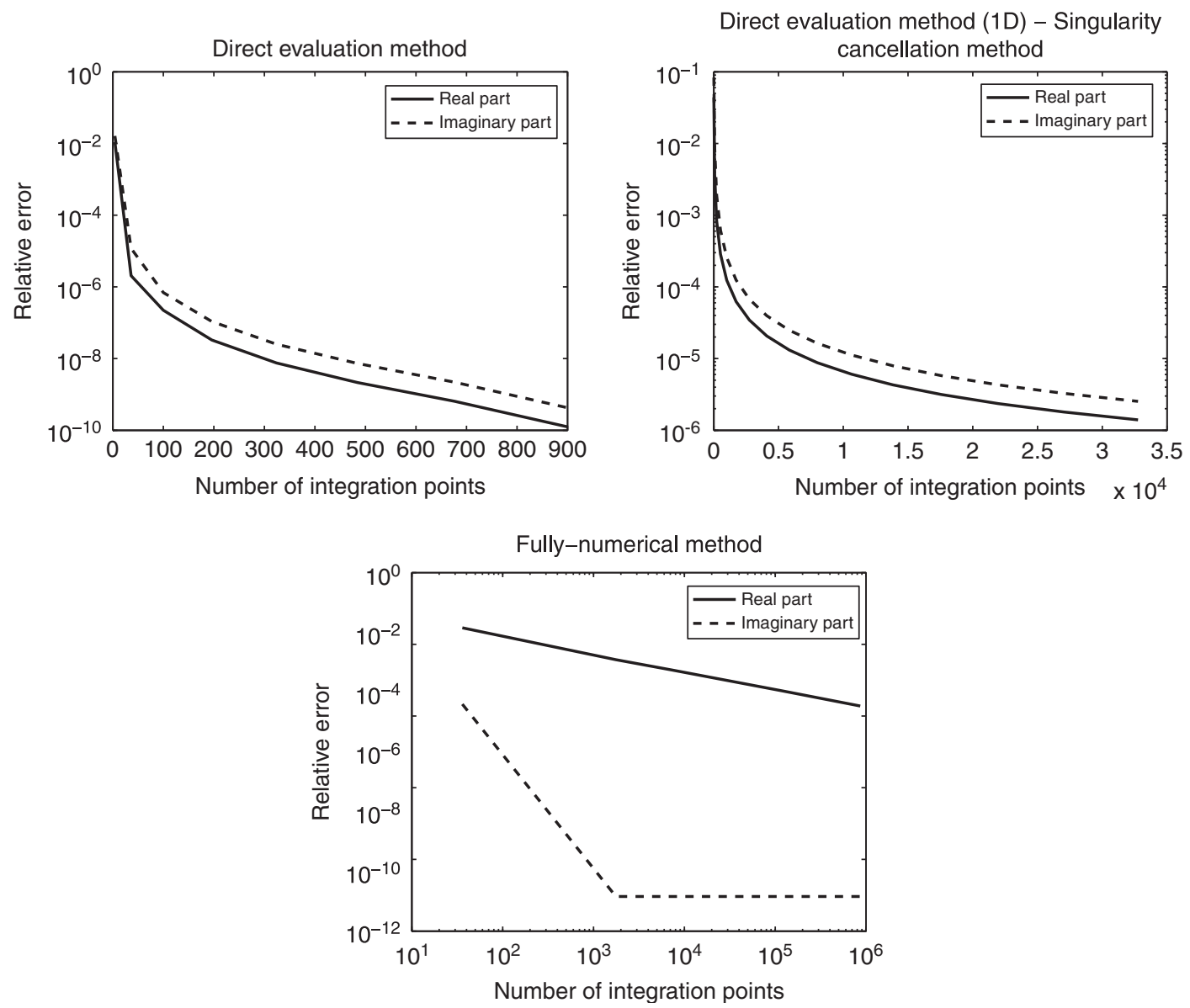

Figure 9. Relative error in calculating the real and the imaginary part of the weakly singular integral $I_{s}^{\text {sing }}$ over the edge adjacent triangles defined by the vertices: $\boldsymbol{r}_{1}=(1,1,0)$,

$$
\boldsymbol{r}_{2}=(2,1,0), \boldsymbol{r}_{3}=(1,2,0), \boldsymbol{r}_{4}=(1,0,0) \text {. }
$$

Next, in Figure 10 we demonstrate the comparison between the direct evaluation method and the fully numerical method in the computation of the weakly singular integral $I_{2,1}^{\text {sing }}(k=1)$ over the edge adjacent triangles defined by the vertices: $\boldsymbol{r}_{1}=(1,1,0), \boldsymbol{r}_{2}=(2,1,0), \boldsymbol{r}_{3}=(1,2,0), \boldsymbol{r}_{4}=(1,0,0)$. It is clear from the above that the dimensionality reduction in the direct evaluation scheme from 4-D to 2-D leads to a superior performance both in terms of accuracy and efficiency. In other words, the second analytical integration, although not needed in a strictly mathematical sense, is fully justified by the results just presented.

\subsection{Vertex adjacent integration}

At last, in the vertex adjacent case, we proceed in a comparison of the direct evaluation method with the fully numerical scheme. More specifically, in Figures 11 and 12 the relative error in computing 

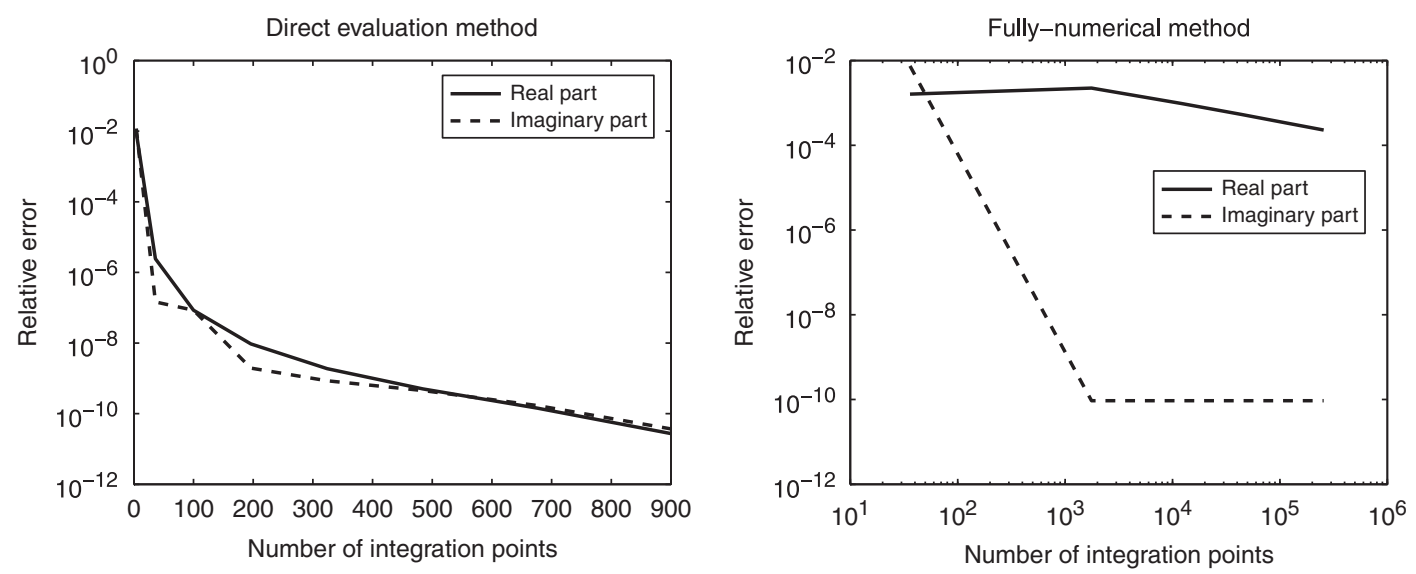

Figure 10. Relative error in calculating the real and the imaginary part of the weakly singular integral $I_{2,1}^{\text {sing }}$ over the edge adjacent triangles defined by the vertices: $\boldsymbol{r}_{1}=(1,1,0)$,

$$
\boldsymbol{r}_{2}=(2,1,0), \boldsymbol{r}_{3}=(1,2,0), \boldsymbol{r}_{4}=(1,0,0) \text {. }
$$
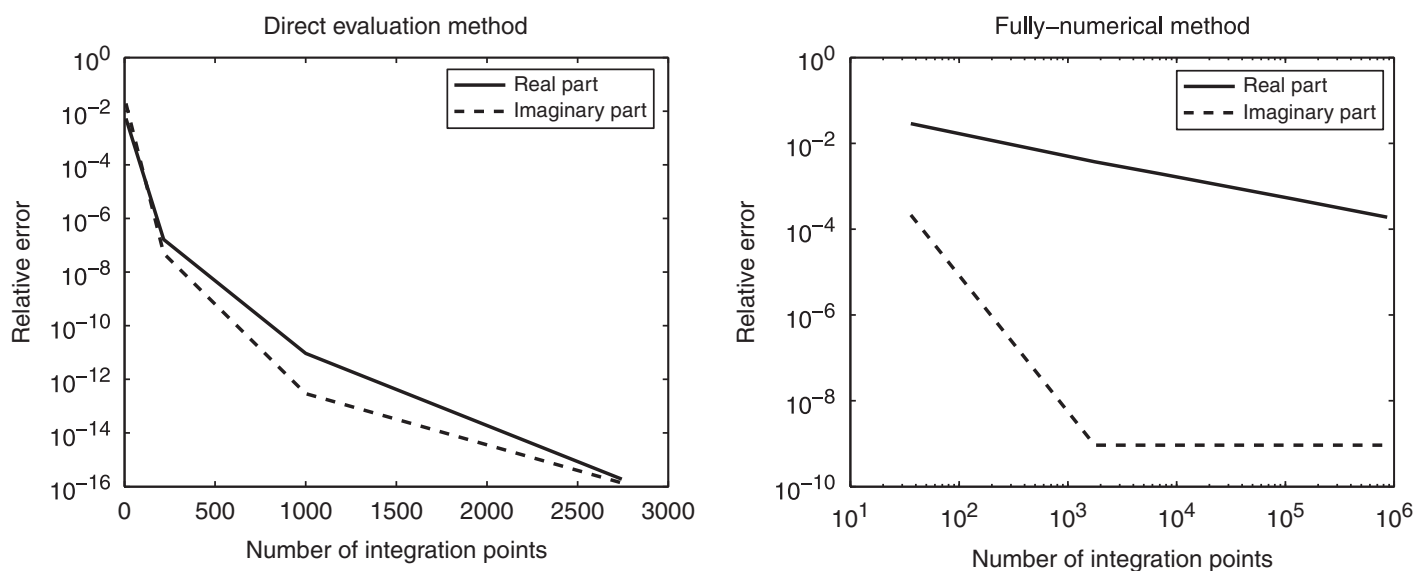

Figure 11. Relative error in calculating the real and the imaginary part of the weakly singular integral $I_{s}^{\text {sing }}$ over the vertex adjacent triangles defined by the vertices: $\boldsymbol{r}_{1}=(1,1,1), \boldsymbol{r}_{2}=(2,1,1)$, $\boldsymbol{r}_{3}=(1,2,1), \boldsymbol{r}_{4}=(0,1,1), \boldsymbol{r}_{5}=(0,2,1)$.

the weakly singular integrals $I_{s}^{\text {sing }}$ and $I_{1,1}^{\text {sing }}(k=1)$ over the vertex adjacent triangles defined by the vertices: $\boldsymbol{r}_{1}=(1,1,1), \boldsymbol{r}_{2}=(2,1,1), \boldsymbol{r}_{3}=(1,2,1), \boldsymbol{r}_{4}=(0,1,1), \boldsymbol{r}_{5}=(0,2,1)$, is shown. Again, as expected, the direct evaluation method succeeds in an accurate evaluation together with a greatly reduced computational burden. Therefore, the treatment of the edge adjacent and vertex adjacent integration with the help of the direct evaluation method is fully justified not only for the presentation of a unified approach (for coincident, edge adjacent and vertex adjacent integrations), but also because of the overall superior behavior in any case and compared with any other method. 

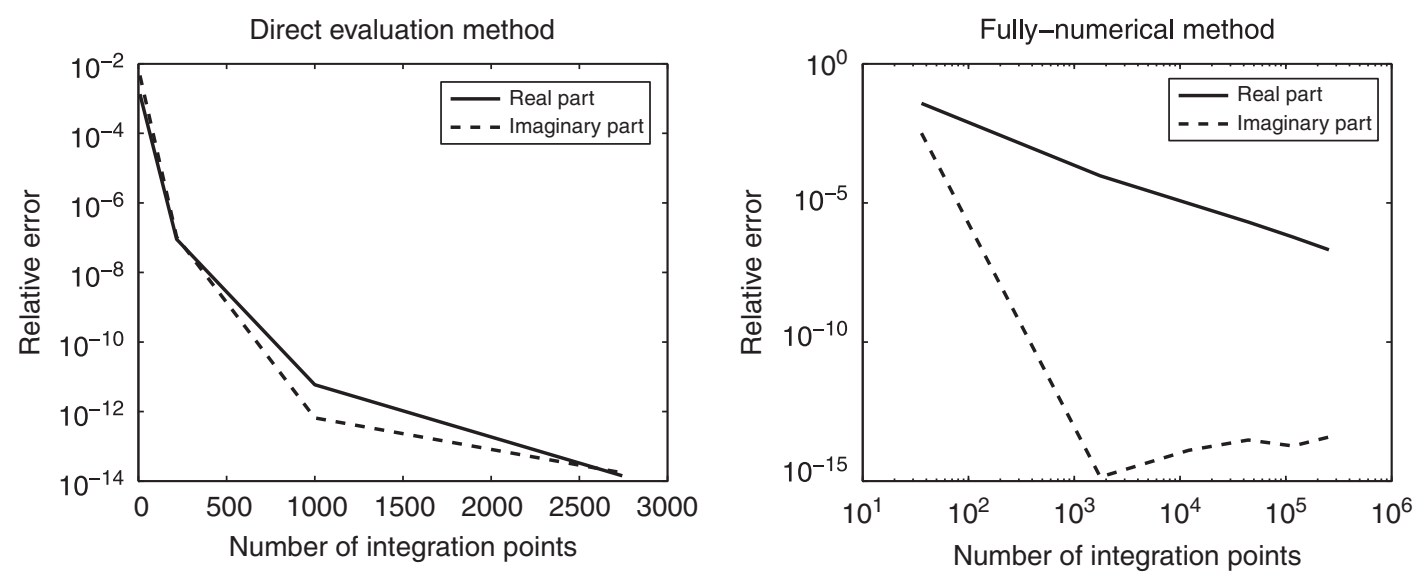

Figure 12. Relative error in calculating the real and the imaginary part of the weakly singular integral $I_{1,1}^{\text {sing }}$ over the vertex adjacent triangles defined by the vertices: $\boldsymbol{r}_{1}=(1,1,1), \boldsymbol{r}_{2}=(2,1,1)$, $\boldsymbol{r}_{3}=(1,2,1), \boldsymbol{r}_{4}=(0,1,1), \boldsymbol{r}_{5}=(0,2,1)$.

\section{CONCLUSIONS}

In this paper we present a complete treatment of weakly singular integrals over coincident, edge adjacent and vertex adjacent triangles arising in the Galerkin discretization of mixed potential integral equation formulations using the linear Rao-Wilton-Glisson basis functions. The numerical experiments undertaken throughout the paper verify the superior performance of the proposed approach in comparison with singularity subtraction, singularity cancellation and fully numerical methods, often used to tackle the weakly singular integration problem. Moreover, the direct evaluation method is expected to be particularly interesting for singular integrations of higher order. Therefore, the detailed analysis presented in this paper forms the backbone for the general treatment of strongly singular and hyper-singular integrals, a very promising course for future work. The benefits of applying the procedures of the direct method to the less singular integrals are primarily the high accuracy, together with a reduced computational work, and a consistent treatment of all integrals.

\section{REFERENCES}

1. Rao SM, Wilton DR, Glisson AW. Electromagnetic scattering by surfaces of arbitrary shape. IEEE Transactions on Antennas and Propagation 1982; 30(5):409-418.

2. Mosig JR, Hall RC, Gardiol FE. Numerical analysis of microstrip patch antennas. In Handbook of Microstrip Antennas, James JR, Hall PS (eds). IEE-Peter Peregrinus: London, 1989.

3. Harrington RF. Field Computation by Moment Methods. Macmillan, Krieger: New York, FL, 1983.

4. Wilton DR, Rao SM, Glisson AW, Schaubert DH, AL-Bundak OM, Butler CM. Potential integrals for uniform and linear source distributions on polygonal and polyhedral domains. IEEE Transactions on Antennas and Propagation 1984; 32(3):276-281.

5. Caorsi S, Moreno D, Sidoti F. Theoretical and numerical treatment of surface integrals involving the free-space Green's function. IEEE Transactions on Antennas and Propagation 1993; 41(9):1296-1301.

6. Graglia RD. On the numerical integration of the linear shape functions times the 3-D Green's function or its gradient on a plane triangle. IEEE Transactions on Antennas and Propagation 1993; 41(10):1448-1455. 
7. Eibert TF, Hansen V. On the calculation of potential integrals for linear source distributions on triangular domains. IEEE Transactions on Antennas and Propagation 1995; 43(12):1499-1502.

8. Hodges RE, Rahmat-Samii Y. The evaluation of MFIE integrals with the use of vector triangle basis functions. Microwave and Optical Technology Letters 1996; 14(1):9-14.

9. Notaros BM, Popovic BD. Optimized entire-domain moment-method analysis of 3D dielectric scatterers. International Journal of Numerical Modelling: Electronic Networks, Devices and Fields 1997; 10:177-192.

10. Bluck MJ, Pocock MD, Walker SP. An accurate method for the calculation of singular integrals arising in timedomain integral equation analysis of electromagnetic scattering. IEEE Transactions on Antennas and Propagation 1997; 45(12):1793-1798.

11. Arcioni P, Bressan M, Perregrini L. On the evaluation of the double surface integrals arising in the application of the boundary integral method to 3-D problems. IEEE Transactions on Microwave Theory and Techniques 1997; 45(3):436-439.

12. Johnston BM, Johnston PR. A comparison of transformation methods for evaluating two-dimensional weakly singular integrals. International Journal for Numerical Methods in Engineering 2003; 56:589-607.

13. Jarvenpää S, Taskinen M, Ylä-Oijala P. Singularity extraction technique for integral equation methods with higher order basis functions on plane triangles and tetrahedra. International Journal for Numerical Methods in Engineering 2003; 56:589-607.

14. Jarvenpää S, Taskinen M, Ylä-Oijala P. Singularity subtraction technique for high-order polynomial vector basis functions on planar triangles. IEEE Transactions on Antennas and Propagation 2006; 54(1):42-49.

15. Telles JF. A self-adaptive co-ordinate transformation for efficient numerical evaluation of general boundary element integrals. International Journal for Numerical Methods in Engineering 1987; 24:959-973.

16. Graglia RD. Static and dynamic potential integrals for linearly varying source distributions in two- and threedimensional problems. IEEE Transactions on Antennas and Propagation 1987; 35(6):662-669.

17. Schwab C, Wendland WL. On numerical cubatures of singular surface integrals in boundary element methods. Numerische Mathematik 1992; 62:343-369.

18. Klees R. Numerical calculation of weakly singular surface integrals. Journal of Geodesy 1996; 70:781-797.

19. Rossi L, Cullen PJ. On the fully numerical evaluation of the linear-shape function times the 3-D Green's function on a plane triangle. IEEE Transactions on Microwave Theory and Techniques 1999; 47(4):398-402.

20. Herschlein A, Hagen Jv, Wiesbeck W. Methods for the evaluation of regular weakly singular and strongly singular surface reaction integrals arising in method of moments. Applied Computational Electromagnetics Society Journal 2002; 17(1):63-73.

21. Cai W, Yu Y, Yuan XC. Singularity treatment and high-order RWG basis functions for integral equations of electromagnetic scattering. International Journal for Numerical Methods in Engineering 2002; 53:31-47.

22. Khayat MA, Wilton DR. Numerical evaluation of singular and near-singular potential integrals. IEEE Transactions on Antennas and Propagation 2005; 53(10):3180-3190.

23. Tang W-H, Gedney SD. An efficient evaluation of near singular surface integrals via the Khayat-Wilton transform. Microwave and Optical Technology Letters 2006; 48(8):1583-1586.

24. Tong MS, Chew WC. Super-hyper singularity treatment for solving 3D electric field integral equations. Microwave and Optical Technology Letters 2007; 49(6):1383-1388.

25. Graglia RD, Lombardi G. Machine precision evaluation of singular and nearly singular potential integrals by use of Gauss quadrature formulas for rational functions. IEEE Transactions on Antennas and Propagation 2008; 56(4):981-998.

26. Taylor DJ. Accurate and efficient numerical integration of weakly singular integrals in Galerkin EFIE solutions. IEEE Transactions on Antennas and Propagation 2003; 51(7):1630-1637.

27. Duffy MG. Quadrature over a pyramid or cube of integrands with a singularity at a vertex. SIAM Journal on Numerical Analysis 1982; 19(6):1260-1262.

28. Gray LJ, Glaeser JM, Kaplan T. Direct evaluation of hypersingular Galerkin surface integrals. SIAM Journal on Scientific Computing 2004; 25(5):1534-1556.

29. Gray LJ, Salvadori A, Phan A-V, Mantic A. Direct evaluation of hypersingular Galerkin surface integrals. II. Electronic Journal of Boundary Elements 2006; 4(3):105-130.

30. Polimeridis AG, Yioultsis TV. On the direct evaluation of weakly singular integrals in Galerkin mixed potential integral equation formulations. IEEE Transactions on Antennas and Propagation 2008; 56(9):3011-3019. 